\title{
Rotating spin-1 bosons in the lowest Landau level
}

\author{
J.W. Reijnders, F.J.M. van Lankvelt and K. Schoutens \\ Institute for Theoretical Physics, University of Amsterdam, Valckenierstraat 65, 1018 XE Amsterdam \\ N. Read \\ Department of Physics, Yale University, P.O. Box 208120, New Haven, CT 06520-8120
}

(Dated: July 11, 2003)

\begin{abstract}
We present results for the ground states of a system of spin-1 bosons in a rotating trap. We focus on the dilute, weakly interacting regime, and restrict the bosons to the quantum states in the lowest Landau level (LLL) in the plane (disc), sphere or torus geometries. We map out parts of the zero temperature phase diagram, using both exact quantum ground states and LLL mean field configurations. For the case of a spin-independent interaction we present exact quantum ground states at angular momentum $L \leq N$. For general values of the interaction parameters, we present mean field studies of general ground states at slow rotation and of lattices of vortices and skyrmions at higher rotation rates. Finally, we discuss quantum Hall liquid states at ultra-high rotation.
\end{abstract}

PACS numbers: 03.75.Mn,05.30.Jp,73.43.Cd

\section{INTRODUCTION}

In recent years, there has been considerable progress in the art of manipulating cold atoms. By varying experimental conditions, a number of quantum states of atomic matter have been realized. In these developments, an exciting theme is the parallel between these states of atomic matter and states of electronic or vortex matter that have been studied before. Two important recent advances are the study of spin-full bosons in optical traps and the analysis of bosons in rotating traps.

Optical traps liberate the (hyperfine) spin degree of freedom of spin-full atoms, as there is no polarizing magnetic field. This allows a variety of new phenomena, such as skyrmions, monopoles and $\pi$-disclinations. There are two different regimes, depending on the sign of the spindependent interaction $c_{2}$. If this interaction is repulsive $\left(c_{2}>0\right)$, the system tends to minimize the total spin and we speak of the anti-ferromagnetic or "polar" regime. If, on the other hand, the interaction is attractive $\left(c_{2}<0\right)$ the system will tend to maximize the total spin. This is the ferromagnetic regime. Examples of such systems are spin-1 Bose-Einstein condensates (BEC) which can be realized by trapping atoms such as $\left.{ }^{87} \mathrm{Rb}\left(c_{2}<0\right)\right)^{1.2}$ and ${ }^{23} \mathrm{Na}\left(c_{2}>0\right)^{\frac{3}{3}}$. In both cases, the ratio $\gamma=c_{2} / c_{0}$ of the spin-dependent $\left(c_{2}\right)$ and the spin-independent $\left(c_{0}\right)$ parts of the (contact) interaction is small, typically a few percent, $|\gamma| \approx 0.01-0.05$.

Rotating the bosons within the trap leads to the formation of quantum ground states with a certain amount of vorticity stored in the system. Upon increasing the rotation rate, a single-component (scalar) condensate with a repulsive interaction goes through the following stages: (i) the nucleation of a single vortex, (ii) the formation of a triangular (Abrikosov) lattice of vortices. Theoretical analysis predicts that this will be followed by (iii), a quantum melting of the lattice and the formation of a (series of) quantum liquids, where the vorticity is spread uniformly over the system. In the presence of internal degrees of freedom, such as those associated with the spin-states of spin-1 atoms, a similar sequence of quantum ground states is expected, with additional structure provided by the internal (spin) degrees of freedom, and by the presence of additional parameters such as $\gamma$.

In this paper we study the ground states of spin-1 atoms in a rotating harmonic trap, focusing on situations where a truncation to the lowest Landau level (LLL) can be justified. We consider generic values of $\gamma$ in the repulsive regime $c_{0}>0$, and also focus on the special case $\gamma=0$, where the interaction has an $S U(3)$ symmetry ${ }^{4}$. Before we come to the details of our analysis, we briefly summarize some of the results in the literature for rotating bosons, with either a single component (scalar case) or several components, such as spin 1 (vector case). Much of this work is based on the restriction to the LLL.

\section{A. Non-rotating condensates and topological excitations}

The case of vector BEC without rotation has been investigated, most often by mean-field theory using the spin-1 Gross-Pitaevskii equations, or with the further approximation of neglecting the kinetic energy term (the Thomas-Fermi approximation). There are two regimes ${ }^{5}$, as mentioned already. In the ferromagnetic regime $c_{2}<$ 0 , the ground state has maximum possible spin, $S=N$. Such a spin state can be constructed by condensing all the bosons into a single-particle state with $S_{z}=+1$, or as a spin-rotation of this. In the opposite antiferromagnetic regime, $c_{2}>0$, the ground state has minimal spin. Such a spin state can be constructed by condensing all the bosons in the same $S_{z}=0$ single-particle spin state, or by taking a spin-rotation of this (notice that this is distinct from all of the ferromagnetic states). This "polar" state breaks spin-rotation symmetry, even though the expectation value of the total spin, or of the spin density, is very close to zero. The distinct ordered states, that 
can be mapped onto each other by the (broken) symmetries of spin rotation and phase rotation, are labelled by points in an order parameter manifold (or target space). For the ferromagnetic case, this manifold is $S O(3)^{5}$, while for the antiferromagnetic or polar case it is $S^{1} \times S^{2} / \mathbb{Z}_{2}{ }^{5.6}$. These ordered states possess excitations that can be described as topological defects in the order, either with a singularity at a point surrounded by a "core", or without a singularity at all. We describe these in more detail in the Appendix, but those that carry non-zero vorticity are relevant to the rotating case which we discuss next.

\section{B. Slow rotation: vortices and skyrmions}

If the number of bosons in a rotating trap is sufficiently large, the effect of slow rotation can be studied in a mean field framework. For a single species of bosons with repulsive interactions, the rotation is accommodated through the creation of singular vortices, with vanishing particle density at the vortex cores. As the rotation rate is increased from zero, there is a critical frequency at which a single vortex first appears in the system, followed by additional vortices at still higher rotation. With a spin degree of freedom, the system has several components in which to store the angular momentum. There is the possibility that a vortex core for one spin component is filled by another spin component, leading to core-less vortices or "skyrmions". In such configurations, the total particle density is nowhere zero, and there is a smooth spin texture. Mean field states of this type for rotating spin1 bosons have been investigated theoretically by solving the spin-full Gross-Pitaevskii (GP) equations 7.8.9.10. For attractive interactions, in contrast, the $\mathrm{BEC}$ remains in a compact blob, without any vortices, all the way up to the maximum rotation frequency (the trap frequency).

\section{Lattices of vortices and skyrmions}

When several vortices are present in a rotating scalar boson condensate with repulsive interactions, they line up in a triangular (Abrikosov) vortex lattice ${ }^{11,12}$. In a vector BEC one expects to find similar lattices, built from the coreless vortices just described. The details of all this depend crucially on the relative strength $\gamma=c_{2} / c_{0}$ of the spin-dependent interaction. For $\gamma=0$, where the $S U(3)$ symmetry between the different spin components is not broken, the lattice that is expected upon rotation is composed of three intertwined triangular lattices. The vortex cores do not overlap, so that the density is (almost) uniform. This lattice has been shown to be independent of the strength of the interaction by Kita et al. 13 . The vortex lattice shows a rich phase diagram, however, when the interaction is spin-dependent. For a range of positive values of $\gamma$, a square lattice composed of $\pi$-disclinations has been predicted ${ }^{13}$.

\section{Scalar boson quantum liquids}

As the rotation increases, quantum fluctuations become more and more important and beyond a critical rotation rate the vortex lattice is expected to melt. The resulting state of matter is disordered, and it has a large amount of vorticity stored in it. In this regime, a number of quantum liquid states have been proposed. Based on the analogy with the physics of electrons in a strong perpendicular magnetic field in two dimensions (2D), the boson quantum liquid states can be characterized as fractional quantum Hall liquids.

An extensive study of the transition to this regime was conducted by Cooper, Wilkin, and Gunn ${ }^{14}$, who performed exact diagonalization studies in the LLL in a toroidal geometry. They predicted that the vortex lattice melts into a quantum disordered phase at a critical value $\nu_{c} \simeq 6-10$ of the filling factor $\nu=N / N_{v}$, with $N_{v}$ the number of vortices and $N$ the number of bosons. They also found that the quantum state is incompressible at $\nu=k / 2<\nu_{c}$, with $k=0,1,2, \ldots$, and observed that the ground states at $\nu=k / 2$ have substantial overlaps with the Read-Rezayi (RR) quantum Hall states ${ }^{15}$.

The RR states are incompressible quantum Hall fluids. The special case $k=1$ is the Laughlin state 16 for bosons at $\nu=1 / 2$, while the $k=2$ state is the Moore-Read "Pfaffian" state 17 . The RR quantum Hall states possess a specific "order- $k$ " clustering property: there is a so-called composite-boson order parameter ${ }^{18.19}$, an operator that creates $k$ bosons and 2 vortices, and is the minimal order parameter (the one that creates the smallest number of bosons) that has long-range ("off-diagonal") order. Such order implies that the quasiparticles over these liquids are (quantized) vortices in the liquid, carrying fractional vorticity $1 / k$, analogous to a fractional magnetic flux $\Phi / \Phi_{0}$ that occurs in certain quantum Hall states in electronic systems. In quantum Hall liquids, the Hall conductivity implies a fundamental quasiparticle charge $q= \pm \nu \Phi / \Phi_{0}$ in units of the charge of the electron. In the context of neutral bosons in a rotating trap, the same argument implies that a fractional particle number $(q= \pm 1 / 2$ for the RR states) is present in the quasiparticle, relative to the background density. Furthermore, for $k>1$ there are non-local degrees of freedom associated with these quasiparticles, that is, the ground states with more than three quasiparticles are degenerate in the limit where all the separations go to infinity. As these degrees of freedom are of a nonlocal, topological nature, they do not couple to local probes and the degeneracy is protected in the large separation limit. For the case $k=2$ there is an interpretation in terms of a Majorana fermion in each vortex $\operatorname{core}^{20}$. Further evidence for the appearance of the Moore-Read state was recently provided by Regnault and Jolicoeur 21 , who observed the low-lying two-particle branch in numerical simulations, upon adding one flux quantum in a spherical geometry. They also found evidence for other quantum Hall states not in the RR series. 


\section{E. Outline}

In previous work ${ }^{4}$ we analyzed spin- 1 bosons in the LLL. We identified attractive and repulsive regimes in the $c_{0}-c_{2}$ plane, and proposed and analyzed two series of clustered quantum Hall states (labeled $S U(4)_{k}$ and $\left.S O(5)_{k}\right)$, analogous to the RR states, for spin-1 bosons in a rapidly rotating trap. We identified the exact ground state for $N$ spin-1 bosons on the disc with one unit of angular momentum per particle, the boson-tripletcondensate (BTC).

In this paper we provide further results on the phase diagram for spin-1 bosons in the LLL. Employing the $S U(3)$ symmetry, we discuss how in a slowly-rotating system with $c_{2}=0$ the exact quantum ground state evolves from the non-rotating one towards the BTC at angular momentum $L=N$, and we compute the ground state angular momentum $L(\omega)$ as a function of the rotation frequency $\omega$. Using LLL-mean field theory, we extend the results for slow rotation to $c_{2} \neq 0$, and discuss the various skyrmion lattices. Furthermore, the two series of quantum Hall states are discussed in detail and we supplement them with a third series.

This paper is organized as follows. In section @ we define the model by discussing LLL truncation in a disc, sphere or torus geometry, specifying the interaction Hamiltonian, and make remarks on the general symmetry properties for later use. In section III we study the phase diagram by direct numerical diagonalization. In section IV] exact quantum ground state wavefunctions and energies for a slowly rotating (angular momentum $L \leq N$ ) system in the $c_{2}=0$ limit are presented. For nonzero $c_{2}$, we use a LLL mean field treatment to study the slowlyrotating system (in section $\nabla$ ) and the various skyrmion and vortex lattices (in section VI). In section VII we discuss the quantum Hall states at ultra-high rotation. In an Appendix we discuss the topological classification of defects.

\section{LLL MODEL HAMILTONIAN AND ITS SYMMETRY}

In this section we describe the truncation of the space of single-particle states to those in the LLL, and then explain the use of different geometries (sphere, torus) once this trunction has been made. Then we give the form of the interaction Hamiltonian that will be assumed, and some analysis of the symmetries of the model, with particular reference to certain limits and different geometries.

\section{A. Truncation to the lowest Landau level}

In a rotating frame of reference the Hamiltonian for $N$ trapped, weakly-interacting spin-1 bosons is

$$
H=\sum_{i}^{N}\left[\frac{\omega_{0}}{2}\left(-\vec{\nabla}_{i}^{2}+r_{i}^{2}\right)-\vec{\omega} \cdot \vec{L}_{i}\right]+H_{\mathrm{int}} .
$$

Here $\vec{\omega}$ is the frequency of the rotation drive, $\vec{L}_{i}$ the angular momentum of the $i$-th particle and $H_{\text {int }}$ the interaction Hamiltonian, which we discuss below. We have set $\hbar$ and the harmonic oscillator length $l \equiv\left(\hbar / m_{b} \omega_{0}\right)^{1 / 2}$ of the trap (with $\omega_{0}$ the trap frequency and $m_{b}$ the boson mass) equal to one. Modes in the direction of the rotation axis are frozen out, leaving us effectively with a two-dimensional (2D) system. The energy eigenvalues of the single-particle part of the Hamiltonian are then $E_{n, m}=(2 n+m+1) \omega_{0}-m \omega$, with $n \geq 0$ the Landau level index and $m \geq-n$ the $z$-component of angular momentum, labelling the states within each Landau level.

We consider the model in which the single-particle states are restricted to the lowest $(n=0)$ Landau level $(L L L){ }^{29}$. This is valid when the interactions are sufficiently weak, as we will explain momentarily. The normalized LLL wavefunctions are $\phi_{m}(z) \zeta^{\alpha}$ with the orbital part $\phi_{m}(z) \propto z^{m} e^{-|z|^{2} / 2}(z=x+i y)$, and $\zeta^{\alpha}$ a threecomponent complex vector representing the spin state; here $\alpha$ labels the eigenstates of the z-component of the spin $S_{z}$ for each particle, $\alpha=\uparrow, 0, \downarrow$. [Later in the paper it will be convenient also to use the basis of Cartesian components for spin 1 , labelled by $\mu=x, y, z$.] When we use second quantization, we will denote the boson creation and annihilation operators for these single-particle states by $b_{m \alpha}^{\dagger}, b_{m \alpha}$, and the corresponding occupation numbers by $n_{m \alpha} \equiv b_{m \alpha}^{\dagger} b_{m \alpha}$. Also, we sometimes use the field operator $\psi_{\alpha}(z)=\sum_{m} b_{m \alpha} \phi_{m}(z)$. The single particle contributions to the Hamiltonian add up to $\left(\omega_{0}-\omega\right) L$, with $L=\sum_{i} L_{z i}$ the $z$-component of total angular momentum. We will refer to this geometry as the disc in view of the form of the fluid states (for repulsive interactions) which tend to form a disc or "pancake", because of the centrifugal force. Note that we must have $\omega \leq \omega_{0}$, otherwise the system becomes unstable.

To study the bulk properties of the quantum ground states, we will eliminate boundary effects by using instead two other geometries and taking the limit $\omega \rightarrow$ $\omega_{0}$. In a spherical geometry ${ }^{22}$, the orbital part of the LLL single-particle wavefunctions is $\phi_{m}(z) \propto z^{m} /(1+$ $\left.\left(\frac{|z|}{2 R}\right)^{2}\right)^{1+N_{v} / 2}$, where $z$ represents position on the sphere by stereographic projection to the plane, and $R$ is the radius of the sphere. The number of orbitals is restricted by the vorticity $N_{v}$ penetrating the sphere, $0 \leq m \leq N_{v}$. The $N_{v}+1$ single-particle orbitals form a representation of orbital angular momentum equal to $N_{v} / 2$, see Ref ${ }^{22}$. In the limit $R \rightarrow \infty$, keeping $N_{v} / R^{2}, N$ and $z$ constant, the single-particle wave functions on the sphere reduce to those for the disc as above. The total angular momentum on the sphere is characterized by quantum numbers 
$\tilde{L}$ for the magnitude, and $\tilde{L}_{z}$ for the $z$-component. In terms of $L$ which has eigenvalues $L=\sum_{i} m_{i}$ as before, $\tilde{L}_{z}=\frac{1}{2} N N_{v}-L$. We emphasize that our definition of $L$ when used for the sphere does not have its usual meaning, but is related to the $z$-component in such a way that the $N_{v} \rightarrow \infty$ limit agrees with the plane.

The final geometry we use is the torus. Here the singleparticle wavefunctions take the form $\phi(z) \propto f(z) e^{-y^{2}}$ in the Landau gauge, with $f$ a quasiperiodic holomorphic function. With $N_{v}$ flux quanta, $f$ has $N_{v}$ zeros in the unit cell. There are exactly $N_{v}$ independent solutions, of the form $f(z)=\prod_{i=1}^{N_{v}} \vartheta_{1}\left(z-z_{i} \mid \tau\right)$, with $\tau$ describing the geometry of the unit cell and $z_{i}$ the zeros of $f$. The use of $\vartheta$-functions ensures that $\phi$ is periodic. Many-body states can be classified by their Haldane momentum 23 .

\section{B. Interaction Hamiltonian}

In a model description, the Hamiltonian describing the 2-body interactions of a system of $N$ spin- 1 bosons is a contact interaction, and contains spin-independent $\left(H_{n}\right)$ and spin-dependent $\left(H_{s}\right)$ terms, of strengths $c_{0}, c_{2}$ respectively

$$
\begin{aligned}
H_{\mathrm{int}} & =H_{n}+H_{s} \\
& =2 \pi \sum_{i<j}^{N} \delta^{(2)}\left(\mathbf{r}_{i}-\mathbf{r}_{j}\right)\left[c_{0}+c_{2} \vec{S}_{i} \cdot \vec{S}_{j}\right] .
\end{aligned}
$$

Here $c_{0}=\left(g_{0}+2 g_{2}\right) / 3, c_{2}=\left(g_{2}-g_{0}\right) / 3, g_{S}=4 \pi \hbar^{2} a_{S} / m_{b}$ and $a_{S}(S=0,2)$ the 2D $s$-wave scattering phase shift in the spin- $S$ channe ${ }^{5,24}$. A factor $2 \pi$ has been extracted for later convenience. One can obtain these parameters by integrating over the third direction. Assuming, for example, harmonic confinement with quantum length $l_{\perp}$ in the $z$-direction, one finds $a_{S}^{2 D}=a_{S}^{3 D} / \sqrt{2 \pi} l_{\perp}$ when $l_{\perp} \ll l$. For the sphere, the coordinates $\mathbf{r}$ in this Hamiltonian take values on the surface of the sphere, with radius $R$.

The use of the LLL reduced Hamiltonian is justified when the interactions are weak. Physical quantities evaluated in the full model differ from those in the LLL model by relatively small corrections when the $\nu c_{S} \ll 2 \omega_{0}$. Here $\nu$ is the typical filling factor (expectation of the occupation numbers, summed over $\alpha$ or $\mu$ ) of the single-particle states. Notice that this condition becomes much less stringent as $\omega \rightarrow \omega_{0}$ in the repulsive regime, as then the particles spread out into a pancake, and the filling factor $\nu$ becomes of order 1 .

Finally then, the LLL Hamiltonian in the rotating frame which we wish to analyze is

$$
H_{\omega}=\left(\omega_{0}-\omega\right) L+H_{\mathrm{int}}
$$

Note that we use precisely this definition in the case of the sphere as well as for the disc. It will be useful also to know the ground states of $H_{\text {int }}$ for each $L$.

\section{C. $S U(3)$ symmetry analysis for $c_{2}=0$}

In general, the only symmetry in spin space of the Hamiltonians $H_{\mathrm{int}}$ and $H_{\omega}$ is spin-rotation symmetry

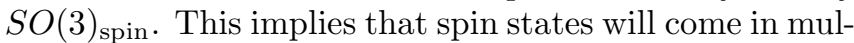
tiplets of spin $S$ with degeneracy $2 S+1$ (with $S$ integer since the particles have spin 1$)$. However, at $c_{2}=0$, the interaction Hamiltonian reduces to the spin-independent interaction $H_{n}$. In this case the spin-rotation symmetry is enlarged from $S O(3)_{\text {spin }}$ to $S U(3)_{\text {spin }}$. It will be useful to understand what this implies about the spin multiplets in a finite size system.

For $c_{2}=0$, the spectrum will contain degenerate spin multiplets labelled by $S U(3)$-quantum numbers $(p, q)$. These tuples are the Dynkin indices labelling irreducible representations of dimension $\operatorname{dim}_{(p, q)}=\frac{1}{2}(p+1)(q+1)(p+$ $q+2)$. Since $S O(3)$ is embedded in $S U(3)$, each multiplet can be decomposed into a set of $S O(3)$ multiplets. These $S O(3)$ spin quantum numbers can be deduced by using branching rules for $S U(3) \mapsto S O(3)$. The fundamental branching rule states that a $(p, 0)$ or $(0, p)$ multiplet contains $S=p, p-2, p-4, \ldots, 1$ (0) for $p$ odd (resp., even). Using the fusion rule

$$
\begin{aligned}
& (p, 0) \otimes(0, q) \\
& \quad=(p, q) \oplus(p-1, q-1) \oplus \cdots \oplus(p-q, 0),
\end{aligned}
$$

which is valid for $p \geq q$, general branching rules can be derived. A multiplet $(p, q)$ with $q$ odd and $p \geq q$ decomposes in $S O(3)$ multiplets with highest weights $S$ according to the branching rule

$$
(p, q) \mapsto \bigoplus_{i=0}^{\frac{q-1}{2}} \bigoplus_{S=2 i+1}^{p+q-2 i} S
$$

For $q$ even we find

$$
\begin{aligned}
& (p, q) \mapsto\left(\bigoplus_{i=0}^{\frac{q-2}{2}} \bigoplus_{S=2 i+1}^{p+q-2 i} S\right) \oplus\left(\bigoplus_{j=\frac{q+1}{2}}^{\frac{p}{2}} 2 j\right), \quad p \text { odd } \\
& (p, q) \mapsto\left(\bigoplus_{i=0}^{\frac{q-2}{2}} \bigoplus_{S=2 i+2}^{p+q-2 i} S\right) \oplus\left(\bigoplus_{j=0}^{\frac{p}{2}} 2 j\right), \quad p \text { even. }
\end{aligned}
$$

Note that the highest $S O(3)$-spin in an $S U(3)$-multiplet $(p, q)$ is always $S=p+q$, and the lowest $S=0$ or 1 .

\section{Orbital symmetry in spherical geometry}

In the plane geometry, $H_{\text {int }}$ is invariant under translations and rotations in the plane. When working on the sphere, this symmetry group is replaced by the rotation group $S O(3)_{\text {orb }}$ (strictly, we should say $S U(2)_{\text {orb }}$ whenever $N_{v}$ is odd) of the sphere. In the limit $R \rightarrow \infty$ described above, this symmetry becomes translations and rotations of the plane. When taking this limit, we also 


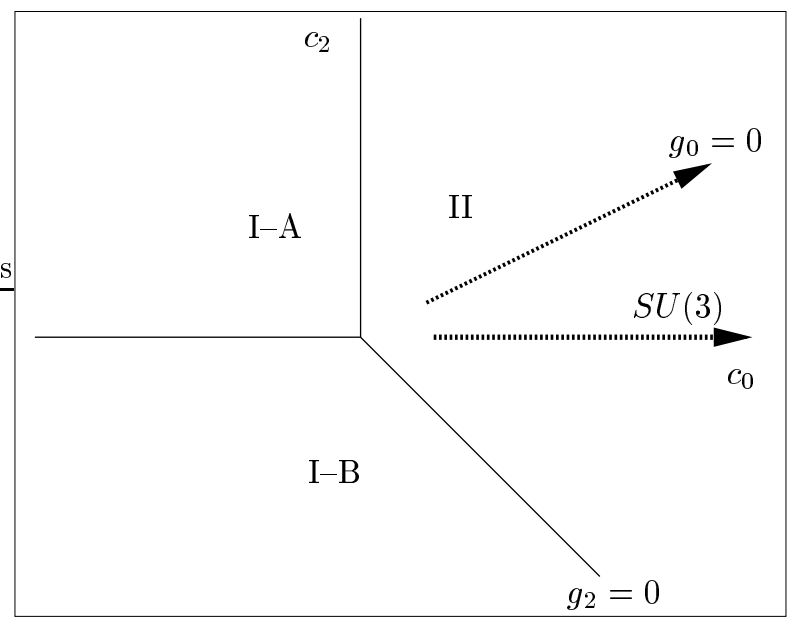

FIG. 1: Overview of $c_{0}-c_{2}$ plane, with special regions and directions marked.

hold $L$ fixed, and hence many-particle states of definite $S O(3)_{\text {orb }}$ quantum numbers $\left(\tilde{L}, \tilde{L}_{z}\right)$ become in the limit infinite-dimensional multiplets of the Euclidean group of the plane. States within each multiplet differ only in the state of the center of mass variable (which has coordinate $\left.z_{c}=\sum_{i} z_{i} / N\right)$. Thus, if $\psi_{L}$ is an eigenfunction of $H_{\text {int }}$ at certain angular momentum $L$, then there exists a whole "tower" of states $\psi_{L+L^{\prime}} \propto z_{c}^{L^{\prime}} \psi_{L}$ with the same interaction energy at angular momentum $L+L^{\prime}$.

We remark that in situations where only a few quantum orbitals are available to the bosons, the spectrum is largely determined by symmetry considerations. Particular examples are the spectrum for $N_{v}=2$ on the sphere, where the exact $N$-body energies are given in terms of Casimir invariants of the orbital and spin-symmetries (see eq. (10) below), and the case with $N_{v}=4$ on the torus, where the topological degeneracy eq. (25) below pertaining to particular quantum liquid states is recovered from the $S U(3)$ spin-symmetry.

\section{MAIN FEATURES OF THE PHASE DIAGRAM}

In this section we make a first pass through the phase diagram with numerical results on moderate sizes. First we consider the ground states of $H_{\text {int }}$ in the disc geometry for each $L$, then use this to find the ground states of $H_{\omega}$ as a function of $\omega$. All this has to be done for general values of $c_{0}, c_{2}$.

\section{A. Global structure of the phase diagram}

First we point out that the magnitude $\left(c_{0}^{2}+c_{2}^{2}\right)^{1 / 2}$ only sets the overall energy scale, so it can be divided out. Thus the phase diagram can be thought of as a circle, in which a point on the circle represents a ray in the $c_{0}-c_{2}$ plane. We wish to examine this for each $L$, or later for each $\omega$. In Figure 1 the $c_{0}-c_{2}$ plane is shown with certain special directions $\left(c_{0}=0, c_{2}=0, g_{0}=0, g_{2}=0\right)$ that will be important later picked out.

For $L=0$, the ground state has total spin $S=N$ for $c_{2}<0$ (ferro regime) and $S=0$ (1) for $N$ even (odd) for $c_{2}>0$ (anti-ferro regime). These states are the way that the broken symmetry states described in Sec. I (the ferromagnetic and polar states respectively) appear in a finite size study. For $c_{2}=0$, there is a single $S U(3)$ multiplet of spin states, decomposing into one $S O(3)$ multiplet of each spin $S=N, N-2, \ldots$ The transition at $c_{2}=0$ can thus be viewed as levels crossing, with a larger degeneracy on the line $c_{2}=0$. As $L$ increases, these two phases at $c_{2} \neq 0$ survive in part of the phase diagram, as compact drops of fluid, with the center of mass carrying all the angular momentum. Meanwhile, the positive $c_{0}$ axis gradually opens into a region that contains other phases. By the time $L$ is $\geq N$, the $c_{0}-c_{2}$ plane contains the three regions labelled $\overline{\mathrm{I}}-\mathrm{A}$, I-B, and II in figure 1

The ground states in regions $\mathrm{I}-\mathrm{A}$ and $\mathrm{I}-\mathrm{B}$ are similar to the one in the "attractive" regime in the scalar case ${ }^{25}$. The orbital part of the ground state wavefunction is of the form $\tilde{\Psi}\left(z_{i}\right) \propto z_{c}^{L}$. In region $\mathrm{I}-\mathrm{A}\left(c_{0}<0, c_{2}>0\right)$, the spin state is the same spin-singlet as for the $L=0$ ground state, and the energy 26 becomes $\left[c_{0} N(N-1) / 2-N c_{2}\right]$. In region $\mathrm{I}-\mathrm{B}\left(c_{2}<0, c_{0}<-c_{2}\right)$, the spin state is ferro, $S=N$, giving energy $25\left(c_{0}+c_{2}\right) N(N-1) / 2<0$. At $c_{2}=$ $0, c_{0}<0$, the spin states again form the $S U(3)$ multiplet. In the remaining "repulsive" region II, the ground state is in general not a common eigenstate of the $c_{0}$ and $c_{2}$ parts of the interaction, and the ground state energy depends non-linearly on the ratio $\gamma=c_{2} / c_{0}$. Note that we have now located the repulsive region more precisely than in our previous characterization of it simply as $c_{0}>0$. Most of the following analysis focuses on region II only, which can be parametrized by $\gamma=c_{2} / c_{0}$ alone.

\section{B. Finite size results in region II as a function of $\omega$}

In figure 2 we show the ground state quantum numbers $(L, S)$ in region II for $N=6$ bosons as a function of the rotation frequency $\omega$. As the phase diagram for each $\omega$ is a circle (which in region II can be parametrized by $\gamma=$ $c_{2} / c_{0}$, or by $\left.\phi=\arctan \gamma\right)$, we are free to plot $\omega$ radially. The parameters are shown in units of $\omega_{0}$ and with $c_{0}=$ 0.25 , but notice that the ground state quantum numbers can only depend on the dimensionless ratios of energies $\left(\omega_{0}-\omega\right) / c_{0}$ and $c_{2} / c_{0}$, so that the structure shown is actually present (though with the radial variable rescaled and shifted) for all parameter values (unless $c_{0}$ is too large). The dashed rays are the lines $c_{2}=-c_{0}, c_{2}=0$ and $c_{2}=c_{0} / 2$, and the outer dashed circle is the locus of $\omega=\omega_{0}$. The ground state angular momentum $L$ and spins $S$ at $c_{2}=0$ and as a function of $\omega$, are shown in the 


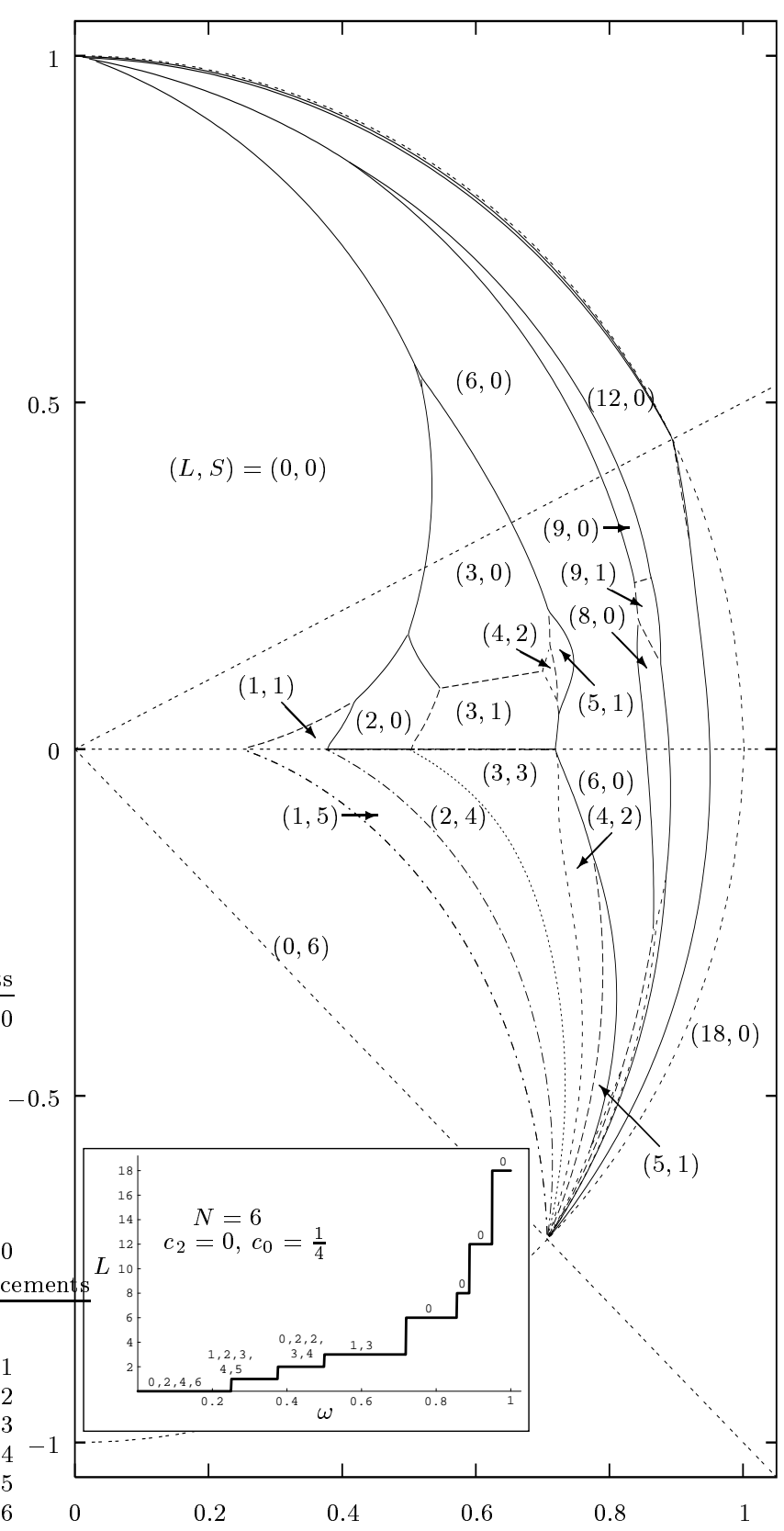

FIG. 2: Ground state quantum numbers $(L, S)$ in region II for $N=6$ spin-1 particles in the planar (disc) geometry, as a function of the driving frequency $\omega$ (plotted radially) and the ratio $\gamma$ (corresponding to the angle with respect to the horizontal axis). The special directions $g_{2}=0, c_{2}=0, g_{0}=0$, $c_{0}=0$ are shown as double-dotted radial lines. The inset shows a cut along the $c_{2}=0$ direction, with the angular momentum given on the vertical axis and the (degenerate) spin values $S$ marked at each of the steps. In this figure, the parameters $c_{0}, c_{2}$, and $\omega$ are in units of $\omega_{0}$, and the value $c_{0}=0.25$ is used in the main figure as well as in the inset. For additional discussion, see the main text. inset. The degenerate spin values at $L \leq N$ are seen to correspond to the following irreducible $S \bar{U}(3)$ multiplets: $(p, q)=(6,0)$ for $L=0,(p, q)=(4,1)$ for $L=1,(p, q)=$ $(2,2)$ for $L=2,(p, q)=(1,1)$ for $L=3$ and $(p, q)=$ $(0,0)$ for $L=6$. Note also that for $c_{2}<0, c_{0}>-c_{2}$ the ground state spin gradually decreases from $S=6$ at $\omega=0$ to $S=0$.

\section{SLOW ROTATION: EXACT GROUND STATES AT $c_{2}=0, L \leq N$}

For larger sizes, a brute-force numerical approach is not feasible, so we develop other approaches. In this section we determine the exact ground state energies and wavefunctions for slow rotation (angular momentum up to the boson number, $L \leq N$ ), for $c_{0}>0$ and $c_{2}=0$, exploiting the $S U(3)$ symmetry described in the section Some of the ground states we find were described in Ref ${ }^{27}$. We analyze a system of $N$ spin- 1 bosons in spherical geometry with $N_{v}$ quanta of vorticity, with the disc geometry emerging as the limit $N_{v} \rightarrow \infty$. We remark that for $N$ sufficiently large, it becomes natural to discuss low energy properties in terms of mean field configurations that break the various symmetries and whose energy is slightly higher than that of the exact quantum ground state; this will be discussed in section $\nabla$

\section{A. Exact eigenstates of $H_{n}$}

The ground state spectrum for $c_{2}=0$ and $L \leq N$ can be understood by exploiting the $S U(3)$ symmetry of the Hamiltonian $H_{n}$. In our analysis we proceed as follows. We consider two series of eigenstates of $H_{n}$, in which (roughly speaking) the bosons occupy at most the lowest three orbitals. Among these eigenstates, we identify the exact quantum ground states on the disc and the sphere, as a function of the angular momentum. This then allows us to compute the $\omega$ dependence of the ground state angular momentum for general $N$ at $c_{2}=0$.

We write the first series of eigenstates as $|p, q, n\rangle^{I}$. These states contain doublets and triplets of spin-1 bosons that are fully antisymmetric in spin indices, and in the orbital indices (guaranteeing the overall symmetry that is required). The different numbers of single bosons, doublets and triplets correspond uniquely to the values of $N$ and the quantum numbers $(p, q)$ of the corresponding $S U(3)$ multiplets. The triplets, which appear $n$ times, are singlets under $S U(3)$, and so do not affect the overall $S U(3)$ representation. The highest spin component $\left(S^{z}=p+q\right)$ of the corresponding $S U(3)$ multiplet takes the following form (up to normalization)

$$
\begin{aligned}
& |p, q, n\rangle^{I} \propto \\
& \quad\left[\vec{e}_{1} \cdot \vec{B}_{\uparrow}^{\dagger}\right]^{p}\left[\vec{e}_{2} \cdot\left(\vec{B}_{\uparrow}^{\dagger} \times \overrightarrow{B_{0}^{\dagger}}\right)\right]^{q}\left[\vec{B}_{0}^{\dagger} \cdot\left(\vec{B}_{\uparrow}^{\dagger} \times \vec{B}_{\downarrow}^{\dagger}\right)\right]^{n}|0\rangle,
\end{aligned}
$$

with $\vec{e}_{1}=(1,0,0), \quad \vec{e}_{2}=(0,0,1)$ and $\vec{B}_{\alpha}^{\dagger}=$ 
$\left(b_{0, \alpha}^{\dagger}, b_{1, \alpha}^{\dagger}, b_{2, \alpha}^{\dagger}\right)$. Clearly, the total number of bosons is $N=p+2 q+3 n$. The energies corresponding to eq. (8) are

$$
\begin{aligned}
E_{p, q, n}^{I} / c_{0}= & \alpha_{1}^{N_{v}} n(n-1)+\alpha_{2}^{N_{v}} q(q-1)+\frac{1}{2} p(p-1) \\
& +\alpha_{3}^{N_{v}} n p+\frac{3}{2} q p+\alpha_{4}^{N_{v}} n q
\end{aligned}
$$

with

$$
\begin{gathered}
\alpha_{1}^{N_{v}}=3 \frac{11 N_{v}^{2}-20 N_{v}+6}{4\left(2 N_{v}-3\right)\left(2 N_{v}-1\right)} \quad \alpha_{2}^{N_{v}}=\frac{5 N_{v}-2}{2\left(2 N_{v}-1\right)} \\
\alpha_{3}^{N_{v}}=\frac{7 N_{v}-4}{2\left(2 N_{v}-1\right)} \quad \alpha_{4}^{N_{v}}=\frac{5 \alpha_{2}^{N_{v}}}{2} .
\end{gathered}
$$

This energy is for spherical geometry, and it depends on the number $N_{v}$ of flux quanta. For $N_{v} \rightarrow \infty$ eq. (9) gives the energy in a disc geometry; $N_{v}=2$ gives the energy on a sphere with 3 orbitals. On the basis of exact diagonalization studies for $N=6,9,12,15,18$ particles we claim that on the disc for $L \leq N / 2$, the ground state multiplet is precisely $|p, q, 0\rangle^{I}$, with $p=N-2 L, q=L$.

On the sphere with $N_{v}=2$, we have obtained a much stronger result ${ }^{28}$, namely a closed form result for all eigenvalues of $H_{n}$. It turns out that these energies can be given in terms of the number $N$ of bosons, the total angular momentum $\tilde{L}$ and the $(p, q)$ labels of the $S U(3)$ representation, according to

$$
E_{p, q}^{N_{v}=2} / c_{0}=\frac{5}{18} N(N-1)+\frac{1}{6} T_{p, q}^{2}+\frac{1}{6} \tilde{L}(\tilde{L}+1),
$$

where $T_{p, q}^{2}=\left(p^{2}+q^{2}+p q\right) / 3+p+q$ is the quadratic Casimir operator for $S U(3)$ in the representation $(p, q)$. Specializing this expression to the states in series I, by eliminating $N$ in favor of $n$ and using the fact that $\tilde{L}=$ $p+q$, reproduces the result in eq. (9) for $N_{v}=2$.

Analyzing the ground state on the disc for $L>N / 2$, we identified a second series of states $|p, q, n\rangle^{I I}$. One can think of the type II states as having the $p$ single bosons in $m=1$ rather then $m=0$, so that now $\vec{e}_{1}=(0,1,0)$. That is not quite correct for the energy eigenstates, as we will explain below, but it does give the correct quantum numbers. The states in series I, II share the property of having $p$ single bosons and $q$ doublets, leading to $S U(3)$ Dynkin labels $(p, q)$. It may be illuminating to display the structure of the states in terms of diagrams similar to Young tableaux as in figure 3 For the orbital structure of the highest-weight states in either series I or II, the lengths of the three rows represent the number of bosons in the orbitals $m=0,1,2$ respectively (in the rough point of view, which will be corrected below), while the differences $p, q$ and $n$ in the lengths correspond to the $S U(3)$ structure. Essentially, these diagrams are ordinary Young tableaux for the states, but with the first two rows exchanged in the case of series II.

For the case of the type II states, the following correction must be made to obtain the energy eigenstates. In the case of scalar bosons, it is known ${ }^{29.30 .31}$ that the
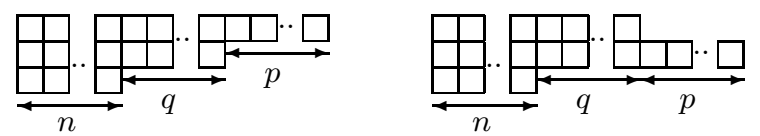

$|p, q, n\rangle^{I}$

$|p, q, n\rangle^{I I}$

FIG. 3: The structure of the two different series of eigenstates of $H_{n}$, displayed in a form similar to Young tableaux. In both cases, the corresponding $S U(3)$-representation has Dynkin labels $(p, q)$.

ground state configuration at $L=p$ of $p$ bosons is a vortex located at their center of mass, with wavefunction $\prod_{i}\left(z_{i}-z_{c}\right)$ with $z_{c}=\sum_{i} z_{i} / p$. This state is not entirely restricted to the $m=1$ orbital, as there are components in which other orbitals in the range $0 \leq m \leq p$ are occupied as well. The $p$ bosons in the state $|p, q, n\rangle^{I I}$ form such a vortex. This complication makes it difficult to write down the closed form expression for the states in series II; based on numerical analysis for small $N$ and mean field results for large $N$ (see section $\nabla$ ), we do propose the following closed form expression for the corresponding energy on the disc

$$
\begin{aligned}
E_{p, q, n}^{I I} / c_{0} & =\frac{33}{16} n(n-1)+\frac{5}{4} q(q-1)+\frac{1}{4} p(p-2) \\
& +\frac{25}{8} n q+\frac{11}{8} n p+q p .
\end{aligned}
$$

Note that the $p$-independent terms in this formula are identical to those for type I states with $N_{v}=\infty$. The state $|p, 0,0\rangle^{I I}$, has energy $p(p-2) / 4$, which is exactly the ground state energy of a rotating scalar BEC at $L=$ $p=N$. This justifies the interpretation of the polarized subsystem with $p$ bosons forming a vortex at the center of mass. However, it turns out that $|p, 0,0\rangle^{I I}$ will never be the lowest energy configuration for a rotating spin-1 system.

Among the type I/II states the following are special. First, $|p, 0,0\rangle^{I}$ is the non-rotating ground state, corresponding to the $(p, 0)$-multiplet. Second, $|0, q, 0\rangle$ gives a wavefunction composed of anti-symmetrized pairs of bosons, a Boson-Doublet-Condensate (BDC) or $(0, q)$ multiplet. Third, $|0,0, n\rangle$ is composed of 3-body singlets. It is a condensate of triplets or boson-triplet-condensate ${ }^{4}$ (BTC); we shall see that it forms the ground state at $L=3 n=N$. The BTC-state can be regarded as a symmetrized version of the core-less vortices observed in mean field studies (see section $\nabla$ for more on this).

More generally, the type I/II states are examples of "(multi-) fragmented" condensates ${ }^{32}$, see also 26 , in the sense that they contain several macroscopically occupied elements in the density matrix. For instance, for the BTC- and for (any component of) the BDC-state we have $\left\langle n_{m \alpha}\right\rangle_{\mathrm{BDC}}=\left(1-\delta_{m, 2}\right)\left(1-\delta_{\alpha \downarrow}\right) q / 2,\left\langle n_{m \alpha}\right\rangle_{\mathrm{BTC}}=$ $n / 3$. Since the spin is fixed in these states, $\left(\Delta n_{\alpha}\right)^{2} \equiv$ $\left\langle\left(n_{\alpha}-\left\langle n_{\alpha}\right\rangle\right)^{2}\right\rangle=0$, where $n_{\alpha}=\sum_{m=0,1,2} n_{m \alpha}$. However, within each spin component, the fluctuations of the boson 


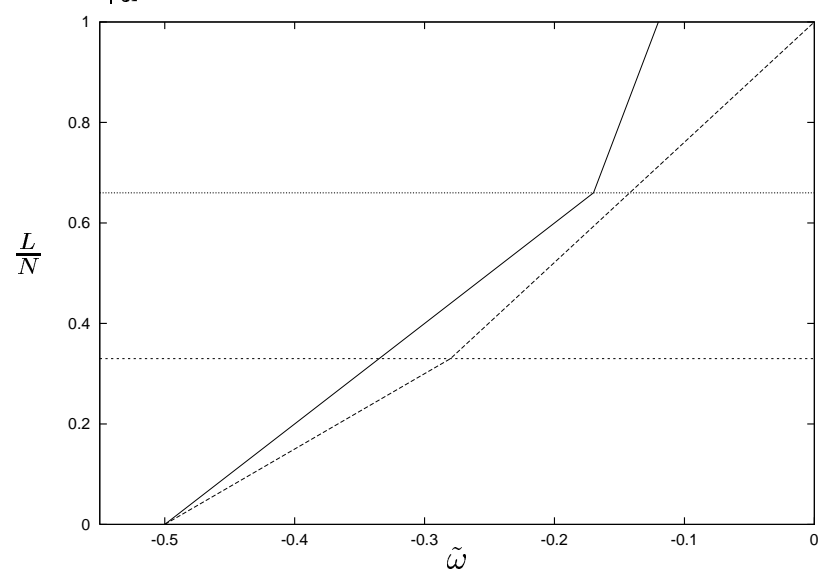

FIG. 4: $L / N$ as $N \rightarrow \infty$ of the ground state on the disc (bold line) and on the sphere with $N_{v}=2$ (dashed line), as functions of $\tilde{\omega}=\left(\omega-\omega_{0}\right) /\left(c_{0} N\right)$ at $c_{2}=0$. The horizontal lines mark the values $L / N=1 / 3$ and $L / N=2 / 3$. The cusps in both curves indicate the point where the $m=2$ quantum orbital is first used in the ground state.

number between orbitals is of the order of the system size: $\left(\Delta n_{m \alpha}\right)_{\mathrm{BDC}}^{2}=q(q+2) / 12,\left(\Delta n_{m \alpha}\right)_{\mathrm{BTC}}^{2}=n(n+3) / 18$. This is an indication that, as in the case of the singlet ground state at $L=0$ in the antiferromagnetic regime and the related "polar" mean field state ${ }^{26}$, it may be best to think of these states as broken symmetry states ${ }^{33}$. That is the approach we will take in Sec. $\nabla$

\section{B. Exact ground states at $c_{2}=0$ as a function of $L$ or $\omega$}

The ground state of a rotating gas with $N$ spin-1 bosons in the LLL and a spin-independent $\left(c_{2}=0\right)$ interaction is formed by a sequence of type I or II states lying on a certain path in $(p, q)$-space as $L$ increases. To find the ground state in a rotating frame of reference, we need to find the ground state of $H_{\omega}$, eq. (3), instead. Since this Hamiltonian contains only two energy scales, the ground state angular momentum per particle $L / N$ can be written as a function of the ratio $\left(\omega-\omega_{0}\right) /\left(N c_{0}\right)$. For finite boson number this function consists of a sequence of steps, as can be seen in figure 2 It turns out that (thanks to our judicious choices of factors of $N$ ) the limit $N \rightarrow \infty$ with $L / N$ and $\left(\omega-\omega_{0}\right) /\left(c_{0} N\right)$ fixed of this function exists, and this is the most convenient information to display. In the following we determine the path of the ground states in $(p, q)$-space as a function of $L$, and the $L(\omega) / N$ behavior of the ground states in this limit for both the sphere $\left(N_{v}=2\right)$ and the disc $\left(N_{v}=\infty\right)$ in the regime $L / N \leq 1$.

\section{Ground states on the sphere at $N_{v}=2$}

On the sphere, our notion of rotation is such that the

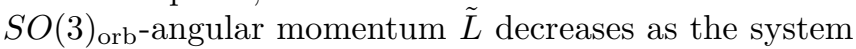
rotates faster and faster. With three orbitals $\left(N_{v}=2\right)$ we have $\tilde{L}_{z}=N-L$ (see section [1A). (We consider $N_{v}=2$ because this case can just accommodate $L \leq$ $N$.) At $\tilde{L}=N$, we know already that the $|N, 0,0\rangle^{I}$ multiplet forms the ground state. As $\tilde{L}$ starts to decrease, again a type I state has the lowest energy; the $(p, q)$ path is parametrized by $(2 \tilde{L}-N, N-\tilde{L})$. Bosons are gradually added to the $m=1$ orbital and form antisymmetrized pairs with the remaining ones. The point up to which this continues can be found by comparing the energies of $|2 \tilde{L}-N, N-\tilde{L}, 0\rangle^{I}$ and $\mid 2 \tilde{L}-N+t, N-$ $\tilde{L}-t, t / 3\rangle^{I}$. After minimizing with respect to $t$ this yields the critical $S U(3)$-indices $(p, q)_{c}=(N / 3, N / 3)$. At this point, with $\tilde{L}=2 N / 3$, ground states with a nonzero $(n>$ 0 ) number of triplets become energetically favorable. In the remaining region, $2 N / 3 \geq \tilde{L} \geq 0$, type I states are the ground states following the path $(p, q)=(\tilde{L} / 2, \tilde{L} / 2)$. Eventually this terminates on the BTC at $\tilde{L}=0 . L / N$ of the ground state as a function of the rotation drive $\omega$ shows a cusp at $\tilde{L} / N=2 / 3(L / N=1 / 3)$, as is shown in figure 4

\section{Ground states on the disc}

For a system on the disc $\left(N_{v}=\infty\right)$, the results are rather different. We will again present the ground states in order of increasing $L$. At $L=0$, the $|N, 0,0\rangle^{I_{-}}$ multiplet forms the ground state as we know. For $L \leq N / 2$ the ground state is formed by a type I state with $n=0$ and $S U(3)$-quantum numbers $(p, q)=(N-2 L, L)$. This state terminates on the $\mathrm{BDC}$ at $L=N / 2$. In this range, increasing $L$ leads, as on the sphere, to more bosons occupying the $m=1$ orbital, forming antisymmetrized pairs with the ones in the $m=0$ orbital. For $L \geq N / 2$, the type II states have the lowest energy. As $L$ increases, bosons move from the $m=0$ into the $m=1$ orbital, decreasing the number of doublets, and giving type II states at $(p, q)=(2 L-N, N-L)$. Comparing the energies of $|t N,(1-t) N / 2,0\rangle^{I I}$ and $\mid(t-s) N,(1-$ t) $N / 2, s N / 3\rangle^{I I}$, we can determine the point where it becomes favourable for triplets to enter the ground state. We find a critical angular momentum $L=\left(1-t_{c}\right) N$ with $t_{c} \sim 1 / 3-3 / N$, which approaches $L=2 N / 3$ for $N$ large. For $L \geq 2 N / 3$ the number of triplets is gradually increasing as $L$ grows. Minimizing $|2 L-N-s, N-L, s / 3\rangle^{I I}$ with respect to $s$, we find that the ground state is now the type II state with $s(N, L)=3 L-2 N$, giving $(p, q, n)=(N-L, N-L, L-2 N / 3)$. For $L=N$ the ground state is the BTC with $p=q=0, n=N / 3$.

To summarize the above results, for $0 \leq L \leq N / 2$ the ground state is given by $|N-2 L, L, 0\rangle^{I}$ and for $N / 2 \leq$ $L \leq 2 N / 3$ by $|2 L-N, N-L, 0\rangle^{I I}$. In the remaining range 
$2 N / 3 \leq L \leq N$ the number of 3-body singlets is nonzero, and the ground state is given by $\mid N-L, N-L, L-$ $2 N / 3\rangle^{I I}$. Minimizing the energy in a rotating frame of reference leads to the $L(\omega) / N$-dependence of the ground states for $N \rightarrow \infty$ which is depicted in figure 4 In this figure, the curve shows a cusp at the point where the $m=2$ orbital first enters the ground state configuration, which is at $L / N=2 / 3$ for the disc with $N$ large. A signature of this cusp in an experimental system might be a change in the expansion rate (the rate of change of the outer radius of the drop with respect to $\omega$ ) if the angular momentum exceeds $2 N / 3$. We shall see that the cusp survives in the anti-ferromagnetic regime, $c_{2}>0$.

It is important to contrast all this with the well-known behavior of scalar bosons in a rotating trap 25.29 . In the latter case there is a jump from $L / N=0$ to $L / N=1$ (for all $N$ ) when one vortex enters the system, whereas for spin-1 bosons we find (at $N \rightarrow \infty$ ) a continuous $L(\omega) / N$ curve with a discontinuous slope.

\section{SLOW ROTATION: LLL MEAN FIELD THEORY}

At low rotation rates, the typical boson occupation numbers $\left\langle n_{m \alpha}\right\rangle$ of the occupied $\left(n_{m \alpha} \neq 0\right)$ single-particle states are large compared with 1 . In this situation, a mean field (or classical) approach to the problem is generally expected to be quantitatively accurate. In such an approach, the boson operators are replaced by expectation values, which are complex c-numbers: $b_{m \alpha}^{\dagger} \rightarrow b_{m \alpha}^{*}$, and the second-quantized Hamiltonian is then minimized with respect to both the magnitude and phase of these numbers to find the ground states. In essence the resulting state is a Bose condensate with the bosons condensed in one linear combination of the single-particle states. This typically involves breaking the orbital and spin symmetries, as well as particle number conservation. (States with definite values of the good quantum numbers such as $N, S, L$ can be obtained afterwards by applying a projection to the mean field quantum state ${ }^{33}$.) In the case of very low rotation, where $L \leq N$, we have seen that (neglecting the subtleties that arose for type II states) the states essentially involve only the $m=0$, 1 , and 2 states, so that the basis set for the mean field calculation is particularly small. In these cases, the mean occupation numbers of the single particle states are of order $N$, and their energies exceed the exact ground state energy (which is of order $N^{2}$ ) by an amount of order $N$. In this section we pursue this mean field calculation for this regime. This gives us easy access to the ground states at large $N$ for $c_{2} \neq 0$ in region II. In the following section, we study instead the mean field states at larger rotation, which can be assumed to be states in which the translational and rotational symmetry group of the plane is broken to that of a lattice.

In terms of the complex numbers $b_{m \alpha}, b_{m \alpha}^{*}$, the energy becomes a quartic polynomial and the ground state can be found by minimizing this polynomial with respect to these variables. This is done here with the mean boson number $\langle N\rangle=\sum_{m \alpha} b_{m \alpha}^{*} b_{m \alpha}$ and angular momentum $\langle L\rangle=\sum_{m \alpha} m b_{m \alpha}^{*} b_{m \alpha}$ fixed at the values $N, L$, respectively. The spin is not constrained at all. For $c_{2}=0$ the Hamiltonian on the sphere takes the form

$$
H_{n}=c_{0} \sum_{\alpha \beta} \sum_{m_{1} \cdots m_{4}} V_{m_{1} m_{2} m_{3} m_{4}} b_{m_{1} \alpha}^{*} b_{m_{2} \beta}^{*} b_{m_{3} \alpha} b_{m_{4} \beta},
$$

with matrix elements

$$
V_{m_{1} \cdots m_{4}}=\frac{1}{2} \frac{\sqrt{\left(\begin{array}{c}
N_{v} \\
m_{1}
\end{array}\right)\left(\begin{array}{c}
N_{v} \\
m_{2}
\end{array}\right)\left(\begin{array}{c}
N_{v} \\
m_{3}
\end{array}\right)\left(\begin{array}{c}
N_{v} \\
m_{4}
\end{array}\right)}}{\left(\begin{array}{c}
2 N_{v} \\
m_{3}+m_{4}
\end{array}\right)} \delta_{m_{3}+m_{4}}^{m_{1}+m_{2}} .
$$

This exhibits the dependence on the spatial orbitals. For $c_{2} \neq 0$, the matrix elements in the additional term consist of $V_{m_{1} \cdots m_{4}}$ multiplied by matrix elements of $\vec{S}_{i} \cdot \vec{S}_{j}$, which depend on the $\alpha_{1}, \ldots, \alpha_{4}$ labels of the bosons. These matrix elements can be found in standard quantummechanics texts.

The fact that mean-field configurations break the various symmetries implies that the minima of the mean field energy form orbits under the action of these same symmetries. On a disc, and at $c_{2} \neq 0$, one expects and finds that, typically, from a generic minimum there are 5 flat directions leading to adjacent minima with equal energy. These flat directions correspond to the 3 generators of the $S O(3)$ spin symmetry, an overall phase, and an orbital $O(2)$ rotation. For spin-independent interactions the symmetry orbits are generically 10 -dimensional.

One convenient quantity to plot is the expectation value $\langle\vec{S}\rangle$ of the spin, whose length is conserved under global spin rotations. In special cases, this expectation value is axi-symmetric; in the more general case it is nonaxisymmetric and the mean field configuration breaks the orbital $O(2)$ symmetry. Another useful quantity is the three-component condensate wavefunction (analogous to the familiar spinor for spin-1/2), which is the expectation value of the field operator, $\left\langle\psi_{\alpha}(z)\right\rangle=\sum_{m} b_{m \alpha} \phi_{m}(z)$ (see Sec. IIA. It is a vector in the $\alpha=\uparrow, 0, \downarrow$ basis. From this we can plot the density in each spin component in position space. This could be accessed experimentally if after switching off the trap to allow the particle cloud to expand, a Zeeman term is switched on, which causes the three $\alpha$ components to separate as they expand.

As an example, we plot in figure 5 the 2D density profile in each spin component of two different mean field ground state configurations at $c_{2}=0, L=N$. The top frame shows the densities for the condensate proportional to $\left(\phi_{0}, \phi_{1}, \phi_{2}\right)$; the lower frame shows a configuration that is related to this by an $S U(3)$ rotation. The total density in each of the $m=0,1,2$ orbitals is an $S U(3)$ invariant, and it is the same for both configurations shown in figure 5 . The mean field energy of these configurations is $E_{M F}=\frac{11}{48} N^{2}$, in agreement with order $N^{2}$ term in the 

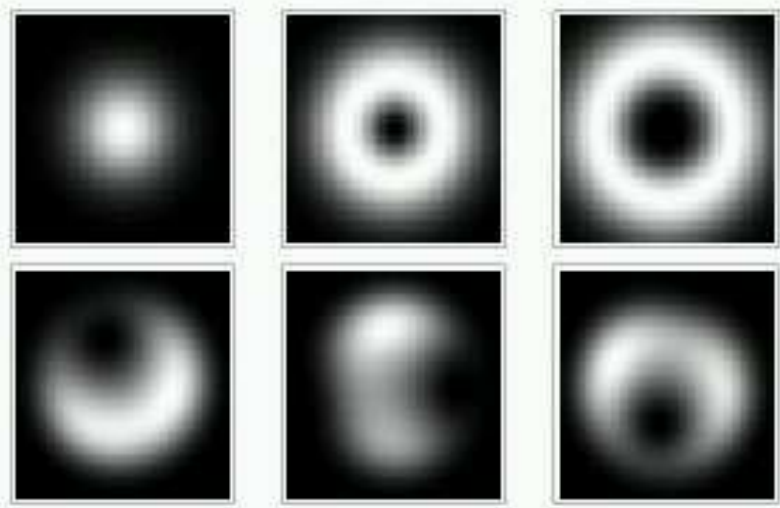

FIG. 5: Two-dimensional density profile of each of the spin components of two LLL mean field ground state configurations at $c_{2}=0, L=N$. The upper panels shows the axi-symmetric spin-vector configuration $\left(\phi_{0}(z), \phi_{1}(z), \phi_{2}(z)\right)$. The two configurations share the same distribution of the total density, and they are related by the $S U(3)$ symmetry. For $c_{2} \neq 0$, there are similarly distinct profiles related by $S O(3)$ symmetry.

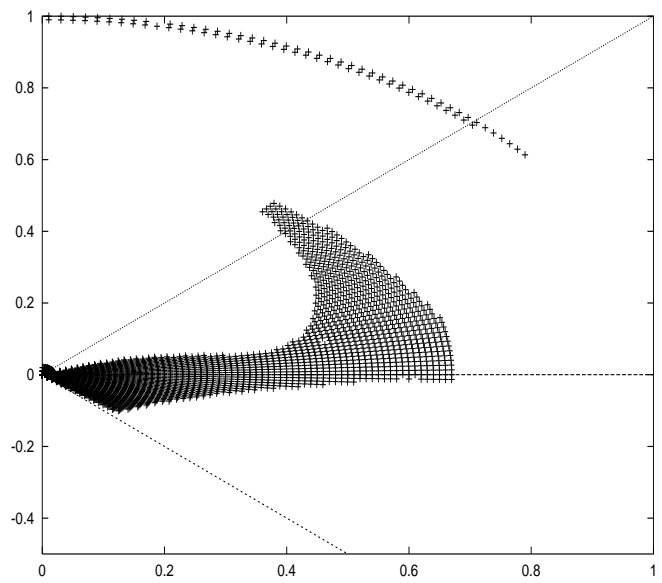

FIG. 6: Regions in the $\gamma, \ell$ plane in which only the $m=0$ and $m=1$ orbitals are present in the mean field ground state on the disc are shaded. The angular coordinate is $\phi=\arctan \gamma$ and $\ell=L / N$ is plotted radially. In the shaded strip near $\ell=1$, a "polar vortex" forms the ground state. The dotted lines mark the $\gamma= \pm 1$ and $\gamma=0$ directions.

energy of the exact quantum (BTC) ground state, eq. (9) with $N_{v}=2, p=q=0$ and $n=N / 3$.

First we consider the disc geometry with $c_{2}=0$. Carrying out the mean field minimizations, we find in terms of $\ell=L / N$ that for $0 \leq \ell \leq 2 / 3$ the number densities $\left\langle n_{m}\right\rangle=\sum_{\alpha} b_{m \alpha}^{*} b_{m \alpha}$ in the orbitals of the mean field ground states behave like (here and in the remainder of this section, these numbers are normalized so that they sum to 1) $\left\langle n_{0}\right\rangle=1-\ell,\left\langle n_{1}\right\rangle=\ell$ and $\left\langle n_{2}\right\rangle=0$. For $\frac{2}{3} \leq \ell \leq 1$ we find $\left\langle n_{0}\right\rangle=\frac{1}{3},\left\langle n_{1}\right\rangle=\frac{4}{3}-\ell$ and $\left\langle n_{2}\right\rangle=\ell-\frac{2}{3}$.
All this is in agreement with the results derived from the exact quantum ground states in section IV]

For very small interaction ratios $|\gamma| \ll 1$, the total densities in the orbitals remain the same as for $\gamma=0$, but there is non-trivial structure in the spin dependence, leading to spin transitions at critical values of $\ell=L / N$, as we will describe shortly.

In figure [6] we have plotted region II of the phase diagram, this time with $\ell$ radially. The shaded regions show where only the first two orbitals $(m=0,1)$ are present in the condensate. One region is a tiny strip near $\ell=1$ for $\gamma \geq(7+4 \sqrt{2}) / 17 \approx 0.75$, where the $(m, \alpha)=(1,0)$ state is occupied by all the bosons. This state can be seen as a "polar" vortex, since it has the same spin state as the polar BEC. The other region, centered (roughly) around the $c_{2}=0$ axis, contains states in which both the $m=0$ and $m=1$ orbitals are used.

In the anti-ferromagnetic regime for $\ell \leq 1$ there is a large area where the $m=3$ orbital requires a non-zero density; in this area, mean field theory in which only the first three orbitals are used is not valid. However, around and at the $S U(3)$-axis and around the polar vortex as well as in the ferromagnetic regime, the density in the $m=3$ orbital is very small for $\ell \leq 1$ and can safely be ignored. Besides, if the energy $H_{\omega}$ in a rotating frame (see eq. (3)) is minimized, only the states which use the first three orbitals $m=0,1,2$ are of interest for $\ell \leq 1$. [This is with the exception of the vicinities of the boundaries of region II (see figure 11) at $\gamma \rightarrow \infty$ and at $\gamma=-1$.]

In the following subsections we present results for the LLL mean field ground state for $|\gamma| \ll 1$, in both the ferromagnetic and anti-ferromagnetic regimes, and we discuss the ground states at $\ell=1$ for general values of $\gamma$.

Our mean field results pertain to the LLL, relevant for the regime of weak interactions, and they thus differ from the mean field solutions of the GP equations 10.34 . Nevertheless, there is agreement on some of the important features, such as the smooth dependence of $L$ on $\omega$ in the ferro regime, and the role of the state with a single $\pi$-disclination near $\ell=0.5$ in the antiferromagnetic regime ${ }^{34}$.

\section{A. Anti-ferromagnetic interactions}

We now specify the mean field ground states, given in the form of a three-component condensate wave function, for small, positive $\gamma=+\epsilon$, and for $\ell \leq 1$. As before, the condensate wave function is a vector in the $\alpha=\uparrow, 0, \downarrow$ basis. In the table below we specify the mean occupation numbers of the four states that we found.

Note that the condensates given in this table are specific representatives of families of condensates that are related by the $S O(3)_{\text {spin }}$ symmetry. There are two critical values, $\ell_{a}^{\epsilon}=\frac{4}{7}-\frac{\sqrt{2}}{7} \approx 0.37$ and $\ell_{b}^{\epsilon}=10-4 \sqrt{5}-$ $\frac{4}{3} \sqrt{85-38 \sqrt{5}} \approx 0.83$, where we see a discontinuous rearrangement of the condensate configuration and of $\langle\vec{S}\rangle$. 


\begin{tabular}{|c|c|c|c|c|c|c|}
\hline & $\left\langle n_{0 \uparrow}\right\rangle$ & $\left\langle n_{0 \downarrow}\right\rangle$ & $\left\langle n_{10}\right\rangle$ & $\left\langle n_{1 \uparrow}\right\rangle$ & $\left\langle n_{20}\right\rangle$ & $\left\langle n_{2 \uparrow}\right\rangle$ \\
\hline $0 \leq \ell \leq \ell_{a}^{\epsilon}$ & $\frac{1}{2}(1-\ell)$ & $\frac{1}{2}(1-\ell)$ & $\ell$ & 0 & 0 & 0 \\
\hline$\ell_{a}^{\epsilon} \leq \ell \leq \frac{2}{3}$ & 0 & $1-\ell$ & 0 & $\ell$ & 0 & 0 \\
\hline$\frac{2}{3} \leq \ell \leq \ell_{b}^{\epsilon}$ & 0 & $\frac{1}{3}$ & 0 & $\frac{4}{3}-\ell$ & $\ell-\frac{2}{3}$ & 0 \\
\hline$\ell_{b}^{\epsilon} \leq \ell \leq 1$ & 0 & $\frac{1}{3}$ & $\frac{4}{3}-\ell$ & 0 & 0 & $\ell-\frac{2}{3}$ \\
\hline
\end{tabular}

TABLE I: Occupation numbers of the LLL mean field ground state with small anti-ferromagnetic interaction $\gamma=\epsilon$.
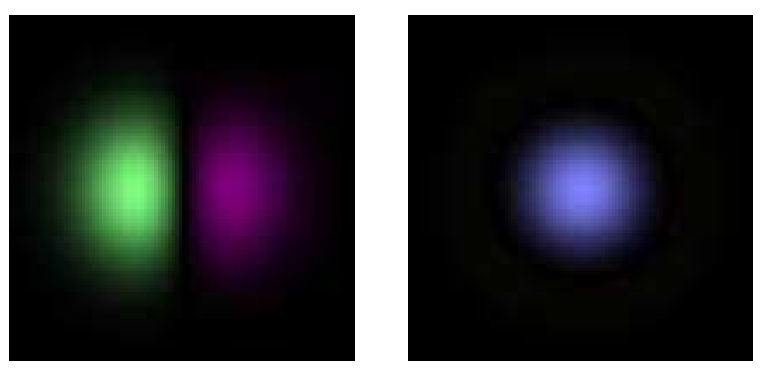

FIG. 7: Two-dimensional plot of $\langle\vec{S}\rangle$ at both sides of the spin transition at $\ell=\ell_{a}^{\epsilon}$. The intensity codes the length $|\langle\vec{S}\rangle|$, while the color indicates the direction on the spin sphere as in figure 10 below. The left and right pictures correspond to eqs. (14) and (15), respectively.

For nonzero $\gamma$, these changes in the condensate are continuous; they become singular (discontinuous) only as $\gamma \rightarrow 0^{+}$.

For $\ell<\ell_{a}^{\epsilon}$, the condensate can be represented by $\left(\frac{\lambda}{\sqrt{2}} \phi_{0}(z), \eta \phi_{1}(z), \frac{\lambda}{\sqrt{2}} \phi_{0}(z)\right)$ with $\lambda=\sqrt{N-L}, \eta=\sqrt{L}$. Applying $S O(3)_{\text {spin }}$ rotations, one finds alternative representations such as $\left(\frac{1}{\sqrt{2}}\left[\lambda \phi_{0}(z)-\eta \phi_{1}(z)\right], 0, \frac{1}{\sqrt{2}}\left[\lambda \phi_{0}(z)+\right.\right.$

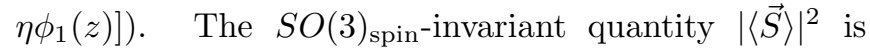
found to be

$$
|\langle\vec{S}\rangle|^{2}=\frac{N^{2}}{\pi^{2}} \ell(1-\ell)(z+\bar{z})^{2} e^{-2|z|^{2}} .
$$

The state that emerges at $\ell>\ell_{a}^{\epsilon}$ corresponds to $\left(\eta \phi_{1}(z), 0, \lambda \phi_{0}(z)\right)$, leading to

$$
|\langle\vec{S}\rangle|^{2}=\frac{N^{2}}{\pi^{2}}\left([1-\ell]-\ell|z|^{2}\right)^{2} e^{-2|z|^{2}} .
$$

For $\ell \neq 1 / 2$, the integrated value of $\langle\vec{S}\rangle$ for this state is non-zero and there is a spontaneous magnetization. In figure 7 a two-dimensional plot of $\langle\vec{S}\rangle$ at both sides of the spin transition at $\ell=\ell_{a}^{\epsilon}$ is shown. The state at $0<\ell<\ell_{a}^{\epsilon}$ can be viewed as a configuration of two $\pi$ disclinations off the center of the trap, while the state in the regime $\ell_{a}^{\epsilon}<\ell<2 / 3$ (or possibly even as far as $\ell_{b}^{\epsilon}$ ) can be understood as a single $\pi$-disclination in the polar state.

The angular momentum for which the $m=2$ orbital is first occupied in the mean field ground state, $\ell=\frac{2}{3}$, is robust against small anti-ferromagnetic interactions.
For $\gamma=+\epsilon, 2 / 3<\ell<\ell_{b}^{\epsilon}$, the condensate can be represented as $\left(\tau \phi_{1}(z), \sigma \phi_{2}(z), \xi \phi_{0}(z)\right)$, with $\xi=\sqrt{\frac{N}{3}}$, $\sigma=\sqrt{L-\frac{2 N}{3}}, \tau=\sqrt{\frac{4 N}{3}-L}$, while for $\ell_{b}^{\epsilon}<\ell \leq 1$ we have $\left(-\sigma \phi_{2}(z), \tau \phi_{1}(z), \xi \phi_{0}(z)\right)$.

In figure 8 we have depicted the ground state angular momentum per particle, $\ell$, as a function of the rotation frequency $\omega$ for some positive values of $\gamma$. It is seen that upon increasing $\gamma$ a semi-plateau (a distinguished part of the curve on which the angular momentum increases gradually) develops. Upon increasing $\gamma$ further, the semiplateau becomes flatter and the width decreases, until for $\gamma$ larger than some critical value $\gamma_{c} \approx 1.19, \ell(\omega)$ jumps from $\ell=0$ to an $\ell=1$ plateau at a critical frequency $\omega_{c}$ given by $\omega_{0}-\omega_{c} \approx 0.15 c_{0} N$. This is a transition from the non-rotating state to the polar vortex, analogous to what occurs in the scalar boson case.

\section{B. Ferromagnetic interactions}

With small negative $\gamma=-\epsilon$ the mean field ground states for slow rotation are characterized (up to $S O(3)_{\text {spin }}$ rotations) by the occupation numbers given in table II Again, we find two spin transitions, the first at $\ell_{a}^{-\epsilon}=$ $2-\sqrt{2} \approx 0.59$ and the second at $\ell_{b}^{-\epsilon} \approx 0.69$.

\begin{tabular}{|l|c|c|c|c|c|}
\hline & $\left\langle n_{00}\right\rangle$ & $\left\langle n_{0 \downarrow}\right\rangle$ & $\left\langle n_{10}\right\rangle$ & $\left\langle n_{1 \downarrow}\right\rangle$ & $\left\langle n_{2 \uparrow}\right\rangle$ \\
\hline $0 \leq \ell \leq \ell_{a}^{-\epsilon}$ & 0 & $1-\ell$ & $\ell$ & 0 & 0 \\
\hline$\ell_{a}^{-\epsilon} \leq \ell \leq \frac{2}{3}$ & $1-\ell$ & 0 & 0 & $\ell$ & 0 \\
\hline$\frac{2}{3} \leq \ell \leq \ell_{b}^{-\epsilon}$ & $\frac{1}{3}$ & 0 & 0 & $\frac{4}{3}-\ell$ & $\ell-\frac{2}{3}$ \\
\hline$\ell_{b}^{-\epsilon} \leq \ell \leq 1$ & 0 & $\frac{1}{3}$ & $\frac{4}{3}-\ell$ & 0 & $\ell-\frac{2}{3}$ \\
\hline
\end{tabular}

TABLE II: Mean occupation numbers of the LLL condensate for small ferromagnetic interaction $\gamma=-\epsilon$.

For $\ell<\ell_{a}^{-\epsilon}$ the condensate can be represented by $\left(0, \eta \phi_{1}(z), \lambda \phi_{0}(z)\right)$ with $\lambda$ and $\eta$ as given above. In this state, the expectation values of the components of the spin vector take the following form

$$
\begin{aligned}
\left\langle S_{x}\right\rangle & =\frac{N}{\pi \sqrt{2}} \sqrt{\ell(1-\ell)}(z+\bar{z}) e^{-|z|^{2}} \\
\left\langle S_{y}\right\rangle & =\frac{N}{\pi \sqrt{2}} \sqrt{\ell(1-\ell)}(-i)(z-\bar{z}) e^{-|z|^{2}} \\
\left\langle S_{z}\right\rangle & =\frac{N}{\pi}(1-\ell) e^{-|z|^{2}} .
\end{aligned}
$$

The state at $\ell_{a}^{-\epsilon}<\ell<2 / 3$ corresponds to $\left(0, \lambda \phi_{0}(z), \eta \phi_{1}(z)\right)$, leading to a spin vector that vanishes at the center of the disc. The spin-textures for $\gamma=-\epsilon$, $\ell<2 / 3$, can be interpreted as half-skyrmions (or merons) (see also section $\mathrm{V}$ D below).

For $\gamma=-\epsilon, 2 / 3<\ell<\ell_{b}^{-\epsilon}$, the condensate can be represented as $\left(\sigma \phi_{2}(z), \xi \phi_{0}(z), \tau \phi_{1}(z)\right)$, with $\xi, \sigma$ 

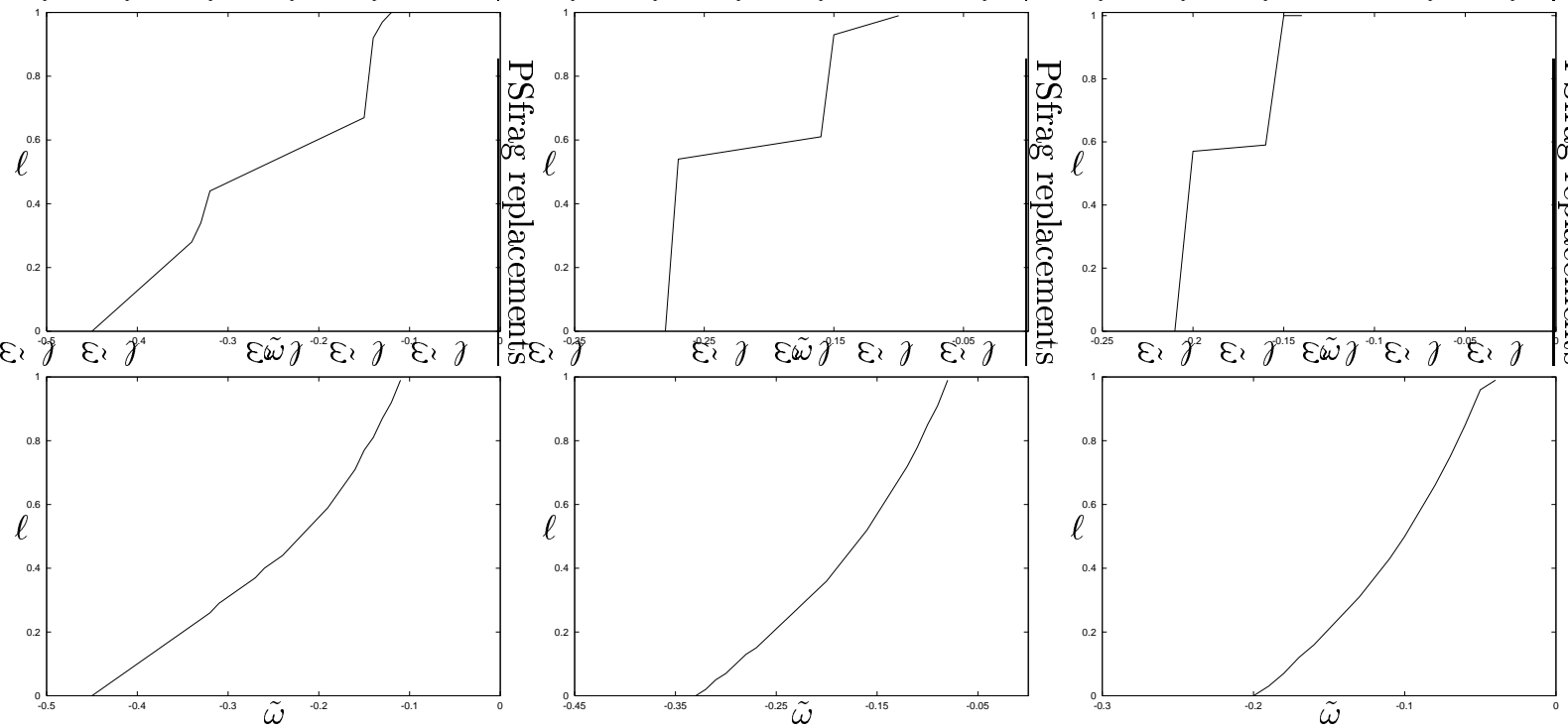

FIG. 8: The ground state angular momentum per particle $\ell$ on the disc as a function of $\tilde{\omega}=\left(\omega-\omega_{0}\right) / c_{0} N$ for various interaction strengths and slow rotation. Upper figures: anti-ferromagnetic regime, $\phi=.1, .5, .75$. Lower figures: ferromagnetic regime, $\phi=-.1,-.3,-.5$.
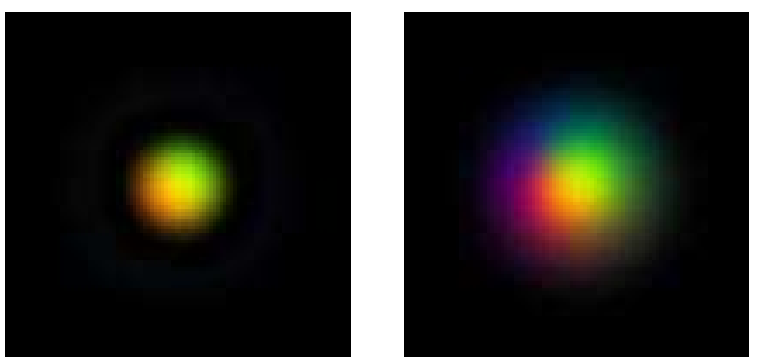

FIG. 9: The spin texture at $l=1$ for $\gamma= \pm \epsilon$. The left side is the anti-ferromagnetic case, the right is the ferromagnetic case. The color coding is as in figure 10

and $\tau$ as above, while for $\ell_{b}^{-\epsilon}<\ell \leq 1$ we have $\left(\sigma \phi_{2}(z), \tau \phi_{1}(z), \xi \phi_{0}(z)\right)$.

In the ferromagnetic regime the $\omega$ dependence of the ground state angular momentum becomes a smooth curve; see Figure 8

\section{Mean field configuration at $L=N$}

Assuming that only the first three $m=0,1,2$ orbitals participate in the ground state, we find that the mean field ground states at $\ell=1$ take the form $\left(\mp \sigma \phi_{2}(z), \tau \phi_{1}(z), \xi \phi_{0}(z)\right)$, with $\xi=\sigma=\sqrt{k_{ \pm}}$and $\tau=\sqrt{1-2 k_{ \pm}}$, and with the $(+)-$sign corresponding to (anti-)ferromagnetic interactions. The parameters $k_{ \pm}$ depend on $\gamma$ according to

$$
k_{ \pm}(\gamma)=\frac{\mp(19+28 \sqrt{2}) \gamma^{2}+(42 \pm 4 \sqrt{2}) \gamma-3}{71 \gamma^{2}+126 \gamma-9} .
$$

The orbital occupation numbers, given as

$$
\left\langle n_{10}\right\rangle=1-2 k_{ \pm}, \quad\left\langle n_{0 \downarrow}\right\rangle=\left\langle n_{2 \uparrow}\right\rangle=k_{ \pm},
$$

are continuous for $\gamma$ going through 0 , but the spintexture, which is sensitive to the phases in the condensate wave function, is not. We find that for $\gamma= \pm \epsilon$, up to an overall constant,

$$
\begin{aligned}
\left\langle S_{x}\right\rangle & =\left(1 \mp \frac{1}{\sqrt{2}} \bar{z} z\right)(z+\bar{z}) e^{-|z|^{2}} \\
\left\langle S_{y}\right\rangle & =\left(1 \mp \frac{1}{\sqrt{2}} \bar{z} z\right)(-i)(z-\bar{z}) e^{-|z|^{2}} \\
\left\langle S_{z}\right\rangle & =\left(1-\frac{1}{2}(\bar{z} z)^{2}\right) e^{-|z|^{2}} .
\end{aligned}
$$

Note that in the anti-ferromagnetic case, the expectation value of the spin vector is vanishing on the circle $\bar{z} z=\sqrt{2}$, while in the ferromagnetic case we see a single skyrmion texture with $\langle\vec{S}\rangle$ non-vanishing everywhere. Figure 9 shows the spin texture at $\ell=1$ for $\gamma= \pm \epsilon$.

From (17) it is possible to derive the critical antiferromagnetic interaction ratio for which the polar vortex appears, by simply solving $k_{+}(\gamma)=0$. The critical value found then is $\gamma^{*}=(7+4 \sqrt{2}) / 17 \approx 0.75$ (see figure 6 ). If $\gamma$ increases towards $\gamma^{*}$, the density in the $m=3$ orbital acquires a small value. So, strictly speaking, the states 
discussed here are not the true mean field ground state in the whole intermediate region. Around $\gamma=0$ and $\gamma=\gamma^{*}$ however, $\left\langle n_{3 \alpha}\right\rangle$ is zero and the value of $\gamma^{*}$ is in agreement with numerical results.

In the ferromagnetic regime, upon lowering $\gamma$ the parameter $k_{-}$gradually decreases from $k_{-}=1 / 3$ at $\gamma=0$ to $k_{-}=1-\frac{1}{\sqrt{2}}$ at $\gamma=-1$, with the corresponding occupation numbers given in eq. (18).

\section{The sphere with $N_{v}=1,2$}

It is instructive to perform LLL mean field theory on a system of spin- 1 bosons in a spherical geometry, with $N_{v}=1$ or $N_{v}=2$, meaning that 2 or 3 orbitals are available to the particles. To compare with the disc as before, we write these results in terms of $\ell=\left(\frac{1}{2} N_{v}-\tilde{L}\right) / N$. Notice, however, that by flattening out the sphere by stereographic projection, the results are qualitatively similar to those for the disc when only the first two or three orbitals are occupied. This is especially true for the states at $N_{v}=2, \ell=1$. Even though the topological classification of textures (see Appendix A) does not strictly apply to the plane, the form of the spin textures on the sphere is a useful guide to those in the disc for $\ell \leq 1$.

For the case $N_{v}=1$ (two orbitals on the sphere), we mention the following results. With $0<\gamma \leq \pi / 4$ the ground state configuration is the same as the one we found on the disc for $\ell<\ell_{a}^{\epsilon}$. This configuration can be interpreted as two $\pi$-disclinations at opposite poles of the sphere. For $\pi / 4 \leq \gamma<\pi / 2$ all bosons occupy the $\alpha=0$ spin component, forming a polar state with a single vortex. In the ferromagnetic regime, with very small $\gamma$ we find the same spin transition as the one on the disc at $\ell=\ell_{a}^{-\epsilon}$. With $N_{v}=1$ this transition lies at $\ell=1 / 2$. These configurations can be interpreted as a half-skyrmion (or meron) in the spin texture. with the spin density vanishing at one point on the sphere, around which the spin density winds around the equator in $\vec{S}$ space, passing over one pole at the opposite end of the sphere. If the interaction is deformed by increasing $N_{v}$ towards $N_{v} \rightarrow \infty$, the location of the spin transition is gradually shifted towards $\ell=\ell_{a}^{-\epsilon}$. With finite ferromagnetic interaction $\left(N_{v}=1\right.$ again), there is a finite region where the core traces a path over the sphere (from the south pole to the north pole, as $\ell$ increases) and connects the two sides of the transition. The interaction energy is clearly independent of $\ell$. This region is bounded by $\gamma(\ell)=-|\arctan (2 \ell-1)|$ for $0 \leq \ell \leq 1$.

For $N_{v}=2$ and $\gamma=0$, the occupation numbers, summed over spin, in the three available orbitals are given by $\left\langle n_{0}\right\rangle=1-\ell,\left\langle n_{1}\right\rangle=\ell$ and $\left\langle n_{2}\right\rangle=0$ for $0 \leq \ell \leq$ $\frac{1}{3}$, followed by $\left\langle n_{0}\right\rangle=\frac{5}{6}-\frac{1}{2} \ell,\left\langle n_{1}\right\rangle=\frac{1}{3},\left\langle n_{2}\right\rangle=\frac{1}{2} \ell-\frac{1}{6}$ for $\frac{1}{3} \leq \ell \leq 1$. These mean field results agree with the exact quantum ground state results obtained in section IV

For $N_{v}=2$ and small ferromagnetic interactions, $\gamma=-\epsilon$, the mean occupation numbers in the conden- sate are given in table (up to $S O(3)_{\text {spin }}$ and $S O(3)_{\text {orb }}$ rotations). In the trajectory from $\ell=0$ to $\ell=1$ there are no spin transitions. The $\ell=1$ state, which has $\left\langle n_{0 \downarrow}\right\rangle=\left\langle n_{10}\right\rangle=\left\langle n_{2 \uparrow}\right\rangle=\frac{1}{3}$, is the mean field ground state for arbitrary ferromagnetic spin interactions, $0>\gamma>-1$. It is a single skyrmion texture with both uniform number density and magnitude of the spin density, and is discussed further in the Appendix.

\begin{tabular}{|c|c|c|c|}
\hline & $\left\langle n_{0 \downarrow}\right\rangle$ & $\left\langle n_{10}\right\rangle$ & $\left\langle n_{2 \uparrow}\right\rangle$ \\
\hline $0 \leq \ell \leq \frac{1}{3}$ & $1-\ell$ & $\ell$ & 0 \\
\hline$\frac{1}{3} \leq \ell \leq 1$ & $\frac{5}{6}-\frac{1}{2} \ell$ & $\frac{1}{3}$ & $\frac{1}{2} \ell-\frac{1}{6}$ \\
\hline
\end{tabular}

TABLE III: Mean occupation numbers of the LLL mean field ground state in spherical geometry, $N_{v}=2$, with small ferromagnetic interaction $\gamma=-\epsilon$.

In the case $N_{v}=2$, and small anti-ferromagnetic interactions, $\gamma=+\epsilon$, for $0 \leq \ell \leq \frac{1}{3}$ the mean occupation numbers per orbital of the condensate are the same as in the ferromagnetic case, but the spin structure is different. For $\ell \geq \frac{1}{3}$ the spin expectation values in the $m=0$ and 2 orbitals become non-zero (without a discontinuity) and are not linear functions of $\ell$. Since $\left|\left\langle\vec{S}_{m=1}\right\rangle\right|^{2}=0$ and the number density is constant in the $m=1$ orbital, the spin state describing the bosons in this orbital can be arranged by an $S O(3)$ rotation to be $(0,1,0) / \sqrt{3}$. The vectors representing the bosons in the $m=0,2$ orbitals then are simply constructed. Together with the previouslymentioned vector they form a mutually orthogonal set which minimizes $H_{n}$. Provided that the spin-vectors are properly normalized, the energy can be expressed in terms of one parameter $\alpha(\ell)$, which is connected to the spin densities by $\cos [2 \alpha(\ell)]=\left|\left\langle\vec{S}_{0}\right\rangle\right| /\left\langle n_{0}\right\rangle=\left|\left\langle\vec{S}_{2}\right\rangle\right| /\left\langle n_{2}\right\rangle$. Minimizing the energy with respect to $\alpha(\ell)$ gives

$$
\alpha(\ell)=\arccos \left[\sqrt{\frac{1}{2}+\frac{\frac{1}{4} \sqrt{\lambda\left(\frac{2}{3}-\lambda\right)}}{1-4 \lambda+6 \lambda^{2}}}\right],
$$

with $\lambda=\frac{1}{2}\left(\ell-\frac{1}{3}\right)$. The maximum of the antiferromagnetic energy is not dependent on the angular momentum and lies at $\alpha=\pi / 2$. In the ferromagnetic case this point minimizes the energy, corresponding exactly to the occupation numbers in table III

At $\ell=1$, there are solutions with uniform density, with an unbroken $S O(3)$ subgroup of the $S O(3)_{\text {orb }} \times S O(3)_{\text {spin }}$ symmetry, as the limiting case of the previous $\ell<1$ states. This case is also discussed in the Appendix. There are also solutions in which the orbital distribution in the mean field configuration of the ground state is not unique. For instance, among the degenerate states at large $\gamma$ we find the polar vortex with $\left\langle n_{10}\right\rangle=1$ and a configuration with $\left\langle n_{20}\right\rangle=\left\langle n_{00}\right\rangle=\frac{1}{2}$. 


\section{E. Comparison with finite size exact states}

It is of interest to try to match the mean field states with ground states found in diagonalization studies, such as those shown in Figure 2 for the disc. Since these have definite values of the quantum numbers, they can be compared with the mean field states only by projecting the latter to components with definite quantum numbers 33 . For each $N$ and $L$, the value of the spin picked out should reflect the form of the interaction, and should presumably be the maximal value in the ferromagnetic regime, and the minimal value in the antiferromagnetic. For the spinindependent case $c_{2}=0$, the lowest $S U(3)_{\text {spin }}$ quantum numbers are favored as ground states.

We will not attempt to identify all the states in Fig.2] in this way, but only some of the more prominent. We have already mentioned that the mean field state at $\ell=1$ and $c_{2}=0$ corresponds to the BTC singlet state. Since the mean field state has equal mean occupation of $(m, \alpha)=$ $(0, \downarrow),(1,0)$, and $(2, \uparrow)$, it does contain a unique singlet component which is exactly the BTC state. When $c_{2}$ is turned on, the quantum numbers remain at $(L, S)=$ $(6,0)$, but the state will be slightly altered in its details. The corresponding skyrmion spin textures on the sphere are also discussed in the Appendix.

The BDC multiplet at $L=N / 2$ for $c_{2}=0$ that uses only $m=0,1$ also deserves comment. This corresponds in mean field theory to the $N_{v}=1$ case discussed above and in the Appendix. When $c_{2}<0$, it becomes a halfskyrmion or meron, which survives for all $-1 \leq \gamma \leq 0$. This meron has no projection to spin 0 , and anyway for this regime maximal spin is expected in the ground state. Indeed, for $N=6$ the corresponding $(L=3)$ state has $S=3$. The whole regime $L / N \leq 1$ for ferromagnetic interactions resembles what one expects for skyrmions, that is $L$ (corresponding to $N-\tilde{L}$ on the sphere) decreasing as $S$ increases, as $S=N-L^{35}$. For $c_{2}>0$ and $L=N / 2$, the lowest-spin part of the BDC state becomes the ground state.

At larger positive $\gamma$, there is a prominent region of $(L, S)=(6,0)$ in the $N=6$ data. At the largest $\gamma$, we expect that this can be identified (in the same sense as the preceding discussion, or as in Ref ${ }^{26}$ ) with the polar vortex state of this section. (In a finite size study, one would not expect to see a transition from the BTC state at $c_{2}=0$ to this polar vortex with the same quantum numbers at large $\gamma$.) The jump from $L=0$ to $L=6$ expected from the mean field is seen in Fig. 2. At smaller $\gamma$, a $(3,0)$ region is seen. We speculate that this state corresponds to a single $\pi$-disclination at the center of the trap (with a second one at infinity, or the opposite pole on the sphere), and that the region corresponds to $\ell_{a}^{\epsilon}<\ell<$ $2 / 3$ in the mean field results. Notice also the prominent semi-plateaus near $\ell=0.5$ in the plots in Fig. 8 at larger $\gamma$.

\section{VORTEX AND SKYRMION LATTICES}

Upon driving the system faster, multiple skyrmions are induced. These are expected to form a lattice and can be well treated in a (quantum Hall) mean field analysis. Such an analysis was performed by Kita et al. 13 , who found a range of different lattices for $c_{2}>0$, depending on the relative strength to $c_{0}$ and the rotation. By including higher Landau levels, they were able to show that some of these lattices are qualitatively identical at high and low rotation. Near $c_{2}=0$, the (scalar) vortex breaks up into three vortices, one for each spin component, forming a triangular lattice. For $c_{2} \geq 0.069 c_{0}$, the vortex splits into two $\pi$-disclinations, which make up a square (anti-ferromagnetic) lattice.

We have carried out a program, similar to Mueller and $\mathrm{Ho}^{36}$, appropriate for a mean field LLL description of a multi-component condensate. The LLL approximation (in the limit $\omega \rightarrow \omega_{0}$ ) fixes the vortex lattice spacing to be equal to the harmonic oscillator length. Note that this is different from the Thomas-Fermi regime, where the distance is fixed by the number of vortices, as the density of bosons is the same as in a non-rotating trap.

Under the assumption that the vortices in each spin component form a Bravais lattice, we can choose the oneparticle wavefunctions to be the torus wavefunctions with $N_{v}$ flux quanta (typically, $N_{v}=1$ or $N_{v}=2$ ). For a scalar condensate, the lattice is completely specified by the geometry $\tau$ of the torus. This wavefunction is periodic up to a gauge transformation, equivalent to requiring $A^{\prime}\left(\mathbf{r}^{\prime}\right)=A(\mathbf{r})$.

In the case of multi-component condensates, however, more general boundary conditions are possible. We only need to demand

$$
\psi^{\prime}\left(\mathbf{r}+\mathbf{L}_{i}\right)=e^{i \Lambda_{i}(\mathbf{r})} U_{i} \psi(\mathbf{r})
$$

where $\mathbf{L}_{i}(i=1,2)$ define the geometry, and $\Lambda_{i}$ is the gauge transformation mentioned above. The matrices $U_{1}$ and $U_{2}$ should commute, $U_{1} U_{2} U_{1}^{-1} U_{2}^{-1}=1$, to obtain single-valued wavefunctions.

We require that $U_{1}$ and $U_{2}$ commute with the Hamiltonian, so that the energy of a unit cell is well-defined. For $\gamma \neq 0$, this implies $U_{i} \in S O(3)_{\text {spin }}$. The common eigenvectors of $U_{1}, U_{2}$ then have eigenvalues $\left(1, e^{i \varphi_{1}}, e^{-i \varphi_{1}}\right)$ and $\left(1, e^{i \varphi_{2}}, e^{-i \varphi_{2}}\right)$. With an overall $S O(3)$ rotation, we can fix the direction of the vector with eigenvalue 1 to be parallel to $\hat{z}$ in spin space. With this, the unit cell of the magnetic order (seen in the spin density which is gauge invariant, for example) is larger than that of the density, but always contains an integer number of the latter.

Using this approach, we can confirm a large part of the phase diagram of Kita et $a^{113}$, but we also find additional phases in the ground states at large $\gamma$. These are polar phases, for which we use a unit cell with a single flux quantum. We will use $\phi=\arctan \gamma$ as the parameter. The minimization procedure uses a simplex downhill algorithm in the geometry $\tau$ and the phases $\varphi_{1}, \varphi_{2}$. The wavefunction is obtained from the polynomial free energy 

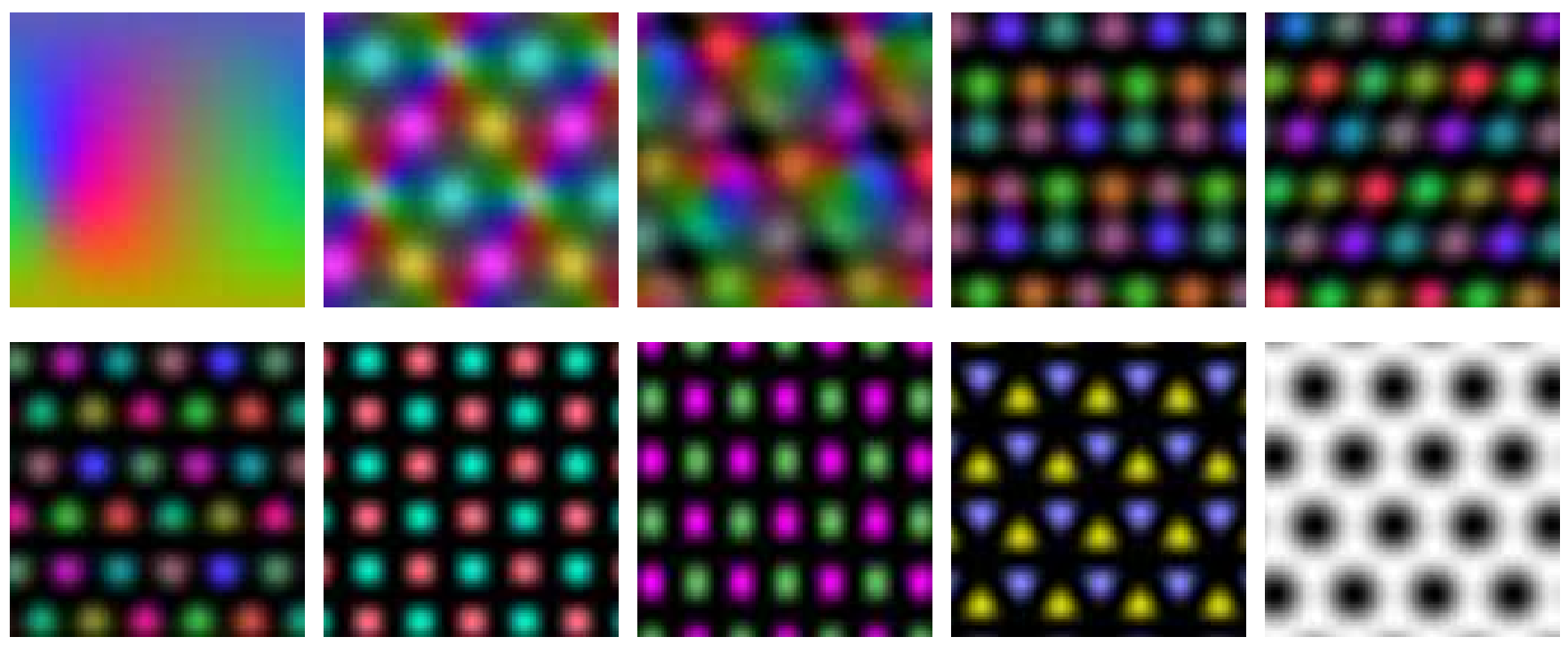

FIG. 10: The different lattices found in rotating spin-1 boson condensates. The first picture is the mapping of the spin-sphere to the colors used. Top and bottom correspond respectively to the north and south pole. The intensity codes $|\langle\vec{S}\rangle|$, the size of the spin-vector. Other pictures are the spin expectations at different ratios $c_{2} / c_{0}: \phi=-0.1,-0.05,0.01,0.016,0.04,0.1,0.54$, 0.7 and 0.9. The last picture shows the density, as the spin vanishes.

by using a conjugate gradient algorithm, starting from a random point. The wavefunction in general is unique up to a phase and a $S O(3)$ rotation along the $\hat{z}$-axis.

The phases we obtain, as illustrated in figure 10, are as follows:

ferro lattice. A major part of the ferromagnetic phase diagram $(-\pi / 4 \leq \phi \leq-0.08)$ is covered by a lattice with $N_{v}=2$ flux quanta in the unit cell. This is the same lattice as one obtains for the spin- $\frac{1}{2}$ bosons with full $S U(2)$ symmetry or, equivalently, the quantum Hall ferromagnet with the Landé factor $g=0$. If we consider the spin- 1 to be composed of two spin- $1 / 2$ particles, then $N_{v}=2$ for the spin- 1 bosons corresponds to $N_{v}=1$ for the spin- $\frac{1}{2}$ particles. This structure is related to the $N_{v}=2$ skyrmions discussed in the Appendix.

skyrmion-vortex lattice. At $\phi \approx-0.08$, it becomes beneficial to include vortices ("merons"). The unit cell now has $N_{v}=3$, with both a skyrmion and a vortex. Based on direct computations in disc geometry (see below), we expect that this phase does not extend to $\phi=0$, but that there are other phases in the weakly ferro regime $-0.02<\phi<0$.

triangular vortex lattice. Exactly at $\phi=0$, the nodes in the three components are arranged in a triangular lattice. This lattice can be realized with $N_{v}=3$ and $\varphi_{1}=\varphi_{2}=0$. The mean-field components $b_{m \mu}$ $(m=0,1,2)$, form a unitary $(U(3))$ matrix. This lattice is not shown in figure 10 as the $S O(3)$-spin is not well defined.

square ladder. The triangular vortex lattice of the $c_{2}=$ 0 case is essentially unchanged up to $\phi=0.0143$, being squeezed only. However, the $S U(3)$ symmetry is broken. This spin shows a ladder structure, where adjacent ladders are shifted by $3 / 2$ rungspacings.

canted ladder, $0.0143 \leq \phi \leq 0.0193$. The ladder structure stays intact, however the rungs are now canted.

triangular ladder, $0.0193 \leq \phi \leq 0.069$.

square $\pi$-disclination, at $\phi \approx 0.069$, there is a first order phase transition to the square $\pi$-disclination lattice. Only the $\uparrow$ and $\downarrow$ components are present in this lattice.

squeezed $\pi$-discl., $0.428 \leq \phi \leq 0.62$. The lattice is squeezed in one direction and expanded in the other.

triangular $\pi$-discl., $0.62 \leq \phi \leq 0.786$. At $\phi \approx 0.62$, there is a first order phase transition to a triangular $\pi$-disclination lattice.

polar Abrikosov, beyond $\phi \approx 0.786$, the $\pi$-disclinations are unstable and the systems prefers to have only one component, such that $\langle\vec{S}\rangle=0 \mathrm{ev}-$ erywhere. The vortices of this component form an Abrikosov lattice, with vanishing density at the cores.

The phases at $\phi>0.428$, and at $\phi<0$ have not been observed before. Figure 10 shows the spin texture in the various lattices, with colors coding the direction of the spin vector and the intensity marking its length, so that black regions indicate places where all components of the spin vector vanish. [For the lattice at $\phi=0.9$, which 
is the polar Abrikosov lattice, the spin density vanishes and we plotted the particle density instead.] The particle density is finite in all lattices except the polar Abrikosov one.

To check whether the Ansatz is sufficiently general in the complete phase diagram, we have supplemented the above analysis by direct numerical computations of LLL mean field ground states in a disc geometry, with $\omega<$ $\omega_{0}$. Since no periodic structure is imposed, the lattices form spontaneously. These computations show that the torus correctly reproduces the dominant phases such as the square lattice of $\pi$-disclinations and the skyrmion and skyrmion-vortex lattices. In the region $-0.02<\phi<0$ the two geometries showed different lattice structures, possibly due to finite size effects. We leave conclusive results in this region for future work.

At special values of $\varphi_{1}, \varphi_{2}$, when they are both of the form $p \pi / q$ ( $p, q$ integer $)$, it is possible to realize the lattice by using a larger unit cell and identical phases for all three spin components. An example of this is the triangular lattice at $c_{2}=0$, where $\varphi_{1}=-\varphi_{2}=2 \pi / 3$. In this case, we can realize the same lattice by using a torus with 3 flux quanta and $\varphi_{1}=\varphi_{2}=0$. The other example is the square $\pi$-disclination lattice, which can be described by using 2 flux quanta. This can be compared to the spin- $1 / 2$ situation $^{36}$, where the lattice at $g_{12}=$ $g_{1}=g_{2}$ (unbroken $S U(2)$ symmetry) can equivalently be described by using a torus with 2 flux quanta 13,37 .

\section{QUANTUM HALL LIQUIDS}

In the LLL approximation, the filling fraction $\nu$ defines the average number of bosons in an orbital. Upon increasing the rotation further, and thus reducing $\nu$, the discreteness of the occupation numbers becomes important. Mean field theory becomes less useful due to quantum fluctuations. At some point, the condensate is destroyed, and a sequence of quantum fluids takes over. In the scalar case, the critical $\nu$ was estimated to be $\nu_{c} \approx 10$ by the Lindemann criterion ${ }^{14}$ (that is, the average fluctuation in the position of the vortices equals the separation between them.) Explicit calculation of small systems have confirmed this transition and found $\nu_{c} \approx 6-10$. A similar transition will occur in the spin-1 case, although we have not calculated the appropriate $\nu_{c}$. We expect this to be of the same order of magnitude as in the scalar case. In the present section we investigate the quantum Hall fluids that appear within region II of the phase diagram in this regime.

In the extreme limit $\omega \rightarrow \omega_{0}(\nu \rightarrow 0)$, we can analyze the quantum liquids analytically in a part of the phase diagram, as we can explicitly find the zero-energy eigenstates of the Hamiltonian. Two of the series we propose, the $S U(4)_{k}$ and the $S O(5)_{k}$ series, have a member of this form for $k=1$. The third series consists of a generalization of a family of fractional quantum Hall (QH) states, the hierarchy/composite fermion states, to spin-1 parti- cles. We present some numerical results on small sizes, which unfortunately are probably not conclusive for the nature of the states, due to the restriction to insufficiently large sizes.

\section{A. $S U(4)_{k}$ series}

It is straightforward to construct zero-energy states at $c_{2}=0$. The repulsive contact interaction dictates that the wavefunction should have a node whenever 2 particles are on the same place. Furthermore, two bosons with the same spin should have a double zero in order to maintain a symmetric wavefunction. In terms of the components of the wavefunction for given values $\mu=x, y, z$ of the spin for each particle, we can write such a state down ${ }^{4,27.38}$ (as in Ref ${ }^{15}$, we add a tilde to the wavefunction to indicate that it has to be multiplied by the usual Gaussian factors for the plane, or the rational factors for the sphere- see Section (II):

$$
\begin{array}{r}
\tilde{\Psi}^{2,2,2,1,1,1}\left(z_{1}^{x}, \ldots, z_{N_{x}}^{x} ; z_{1}^{y}, \ldots, z_{N_{y}}^{y} ; z_{1}^{z}, \ldots, z_{N_{z}}^{z}\right)= \\
\prod_{\mu=x, y, z} \prod_{i<j}\left(z_{i}^{\mu}-z_{j}^{\mu}\right)^{2} \prod_{\mu^{\prime}<\mu^{\prime \prime}} \prod_{i, j}\left(z_{i}^{\mu^{\prime}}-z_{j}^{\mu^{\prime \prime}}\right), \quad(22)
\end{array}
$$

where $n_{\alpha}$ denotes the number of particles with spin $\alpha$. The lowest angular momentum $L$ for which this state can be realized is when $N_{x}=N_{y}=N_{z}=N / 3$. Notice that $L=N(2 N / 3-1)$, and the filling factor, which can be defined as $\nu=\lim _{N \rightarrow \infty} N^{2} /(2 L)$, is $\nu=3 / 4$. This state is a straightforward generalization of both the Laughlin $\nu=1 / 2$ state for spinless particles, and the $(2,2,1)$ Halperin spin-singlet state for spin- $1 / 2$ bosons. The full state for $N_{x}=N_{y}=N_{z}=N / 3$ is an $S U(3)_{\text {spin }}$ singlet $((p, q)=(0,0))$, and arguments similar to Laughlin's plasma mapping show that moreover it has short range spin correlations. Because the state vanishes whenever the particles are at the same point, this state is a zeroenergy eigenstate for all values of $c_{0}, c_{2}$. However, it will only be the ground state (at $L=N(2 N / 3-1)$ ) when $H_{\text {int }}$ is positive, that is in the region $g_{0}, g_{2}>0$, within region II. For larger values of $L$, there are many more zero-energy eigenstates, so the ground states are degenerate in the window $g_{0}, g_{2}>0$. On minimizing $H_{\omega}$ with respect to $L$, this implies that the lowest possible filling factor as $\omega \rightarrow \omega_{0}$ from below is $\nu=3 / 4$ in this regime (within the model in Sec. III).

The state in eq. (22) is an exact eigenstate, but in general we are not able to find the exact highly-correlated ground states of $H_{\text {int }}$. Instead, we seek to understand numerical results, and make predictions for the physics at larger sizes by using (among other techniques) trial wavefunctions. These states, which are not generally exact for any know two-body interaction, serve as paradigms for the phases of matter in the thermodynamic limit, as they possess interesting ("universal") properties such as the quantum numbers and statistics of their excitations that are robust against small changes in the Hamiltonian, 
until some phase boundary is passed (this philosophy has been discussed e.g. in Ref ${ }^{15}$ ). One way to produce trial wavefunctions, that is closely connected to their universal properties, is to use conformal field theory (CFT). We will show how to obtain wavefunctions from a CFT in somewhat more detail in the next section. The CFT describing eq. (22) is $S U(4)_{1}$, so following a strategy in Read-Rezay $1 \frac{15}{15}$, and motivated by the analogous results for scalar bosons ${ }^{14}$, we can consider a series, $S U(4)_{k}$, where $k=1,2, \ldots$ These states have filling factor $\geq 3 / 4$, and are explicitly $S U(3)_{\text {spin }}$ invariant. Hence we expect them to be relevant near $c_{2}=0$.

The trial states we consider have wavefunctions, in spin components (generalizing those in ${ }^{39.40}$ to spin-1),

$$
\tilde{\Psi}\left(\left\{z_{i}\right\}\right)=\mathcal{S}_{\text {groups }} \prod_{\text {groups }} \tilde{\Psi}^{2,2,2,1,1,1} .
$$

In this construction the $N=3 p k$ bosons are first partitioned into $k$ groups, each with $p$ particles of each spin component $x, y, z$.

For each group we write a Halperin $\tilde{\Psi}^{2,2,2,1,1,1}$ factor, and these are multiplied together. Finally, the symmetrization operation $\mathcal{S}$ over all ways of dividing the particles into groups is applied. The angular momentum is $L=N[2 N /(3 k)-1]$, and the filling factor (as $N \rightarrow \infty$ at fixed $k$ ) is therefore $\nu=3 k / 4$. It happens that if we put $k=N / 3$, the state contains $k$ groups of three bosons each, and the state is exactly the $L=N$ BTC. However, we do not believe this is particularly significant, as the BTC state is the unique $S U(3)_{\text {spin }}$ singlet state at $L=N$.

The states (23) are zero-energy eigenstates of a Hamiltonian consisting of a $k+1$-body interaction:

$$
H_{S U(4)_{k}}=V \sum_{i_{1}<\cdots<i_{k+1}} \delta\left(z_{i_{1}}-z_{i_{2}}\right) \cdots \delta\left(z_{i_{k}}-z_{i_{k+1}}\right) .
$$

The interaction is positive for $V>0$, so all eigenstates have $E \geq 0$. This interaction penalizes $k+1$ particles at the same point. Therefore, zero-energy eigenstates are those in which the wavefunction vanishes if any $k+1$ coordinates coincide, regardless of the spins. One can see that the above function has this property, even before the symmetrization over groups, as for any $k+1$ particles, at least two must be in the same group, forcing the function to vanish. For less than $k+1$ particles at the same point, it does not necessarily vanish. In fact, for each $k$, (23) is the unique zero-energy eigenstate of $H_{S U(4)_{k}}$ with lowest angular momentum $L$.

For the same Hamiltonian on a torus, there are again zero energy states, at least for $N$ divisible by $3 k$. For these cases, the degeneracy of these $S U(4)_{k}$ ground states is

$$
\#_{k}=\frac{1}{6}(k+1)(k+2)(k+3) .
$$

We have verified that this result, which can be inferred from the CFT connection, is reproduced by exact diagonalization of the Hamiltonian eq. (24) on the torus.
Like other incompressible $\mathrm{QH}$ states, the phases of matter exemplified by the trial states (23) possess pointlike quasiparticle excitations which may have fractional particle number (relative to the background density) and/or spin. The particle number associated with the elementary quasiparticles can be found once it is understood that, similar to the RR states ${ }^{15}$, the $S U(4)_{k}$ states are clustered states, in which particles occur in clusters

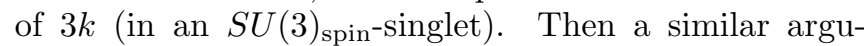
ment to that given in Sec. [shows that they carry charge $q= \pm 1 / 4$. They also have spin 1 . The quasiholes, at which there is a deficiency of particle number, can be studied as zero-energy eigenstates of $H_{S U(4)_{k}}$, and fairly explicit trial wavefunctions can be found using the relation with CFT.

The statistics of quasiparticles in $2 \mathrm{D}$ can be defined in terms of adiabatically dragging them along paths, keeping them well-separated, to exchange them. For the $S U(4)_{k}$ states, in the case $k=1$, the statistics are "Abelian"; the wavefunction acquires a phase factor when two particles are exchanged, just as for the Laughlin states. For $k>1$, however, the statistics becomes "non-Abelian" 15,17. This means that there is a degeneracy, when the positions of the quasiparticles are fixed (in general, this is true only when the quasiparticles are wellseparated, though for the $k+1$-body Hamiltonian and for quasiholes it is exact for any separation). In terms of the trial functions (23) and their generalization to quasiholes, this degeneracy is caused by the symmetrization procedure $\mathcal{S}$, which destroys the "group" quantum number of the (quasi-) particles 39 . The general framework to obtain these degeneracies from CFT has been worked out and agrees with numerical results for the Moore-Read, RR, and other states $17,41.42,43.44$. We expect the same framework to apply here. When the quasiparticles are exchanged adiabatically, the effect is a matrix operation within these spaces of degenerate states, described by the braiding matrices of the corresponding $\mathrm{CFT}^{17}$-hence the term non-Abelian statistics.

\section{B. $S O(5)_{k}$ series}

Similar to the $S U(4)_{k}$ case, the $S O(5)_{k}$ states can be written in the form:

$$
\tilde{\Psi}_{k}^{S O(5)}\left(\left\{z_{i}\right\}\right)=\mathcal{S}_{\text {groups }}\left[P_{\text {groups }} \tilde{\Psi}_{k=1}^{S O(5)}\right],
$$

where now

$$
\tilde{\Psi}_{k=1}^{S O(5)}\left(\left\{z_{i}\right\}\right)=\operatorname{Pf}\left(\frac{\vec{\zeta}_{i} \cdot \vec{\zeta}_{j}}{z_{i}-z_{j}}\right) \prod_{i<j}\left(z_{i}-z_{j}\right) .
$$

Here the spin states for all the particles are included explicitly (the product of spin states $\vec{\zeta}_{i}$ being the tensor product) Pf $M_{i j}$ denotes the Pfaffian of an antisymmetric matrix $M_{i j}$. In the present case the $N=2 k p$ particles are partitioned into $k$ groups, with $2 p$ particles in each. 
The particles in each group form an $S O(3)_{\text {spin }}$ singlet. The product over these groups is then symmetrized. The state as a whole is clearly an $S O(3)_{\text {spin }}$ singlet, and has angular momentum $L=N[N /(2 k)-1]$, so the filling factor is $\nu=k$.

The $k=1$ case, which closely resembles the MooreRead paired state 17 but for spin-1 particles, is the exact ground state of our two-body Hamiltonian $H_{\text {int }}$ when $g_{0}=0$. That is because, in the state $\tilde{\Psi}_{1}^{S O(5)}$, two particles are found at the same point only if they have total spin 0. Again, the ground state as above is the unique zero-energy eigenstate at the stated angular momentum, but at larger $L$ there are many more zero-energy states. So $\nu=1$ is the lowest filling factor possible at $g_{0}=0$. This implies that in finite size on the disc a boundary between ground states with the $L$ values of the $S U(4)_{1}$ and $S O(5)_{1}$ states must run into $\omega=\omega_{0}$ at $g_{0}=0$ (this is for $N$ divisible by 6 , but there will be similar statements for other values). Such behavior is seen in Fig. 2 For $g_{0}<0(\gamma>1 / 2)$, we do not know the exact lowest $\nu$ that occurs as $\omega \rightarrow \omega_{0}$ from below. The $S O(5)_{1}$ state can be interpreted in terms of BCS spin-singlet complex- $-p$ wave pairing of composite fermions, in which the Pfaffian represents the pairing in position space ${ }^{17.20}$.

More generally, for each $k$ there is a Hamiltonian for which the $S O(5)_{k}$ states are exact zero-energy eigenstates, again given by a $k+1$-body interaction:

$$
\begin{aligned}
H_{S O(5)_{k}}= & V \sum_{\substack{i_{1}<\cdots<i_{k}+1\\
}} \delta\left(z_{i_{1}}-z_{i_{2}}\right) \cdots \delta\left(z_{i_{k}}-z_{i_{k+1}}\right) \\
& \times P_{k+1}\left(i_{1}, \ldots, i_{k+1}\right)
\end{aligned}
$$

This interaction includes a projector $P_{k+1}\left(i_{1}, \ldots, i_{k+1}\right)$ of the spin state of the $k+1$ particles concerned onto total spin $k+1$.

For general $k$, these states can be considered to be built up out of clusters of $2 k$ particles in a spin singlet. From this fact we can obtain the fractional particle number of the elementary quasiparticles, $q= \pm 1 / 2$. Also, the quasiparticle spin is $1 / 2$, which is fractionalized compared with the spin 1 of the underlying bosons, and so the number of these quasiparticles must be even. For $k=1$, there are also excitations with zero particle number that behave as fermions with spin 1. In this case, the universal properties may be understood by a simple extension of the methods of Ref ${ }^{20}$ to this case.

Because it is difficult to see through the symmetrization operation $\mathcal{S}$, we will provide some details on the conformal field theory behind these states. Such a CFT description allows us to obtain more insight into the topological properties, such as degeneracies and braiding. For example, to obtain the degeneracy of ground states on the torus, CFT tells us that we only need to know the number of non-trivial representations at level $k$. In the case at hand, this number turns out to be $\frac{1}{2}(k+1)(k+2)$. Again, we verified this number using exact diagonalization of the $(k+1)$-body interaction.

The chiral algebra of the CFT which describes these states is based on the $S O(5)_{k}$ affine Kac-Moody algebra.

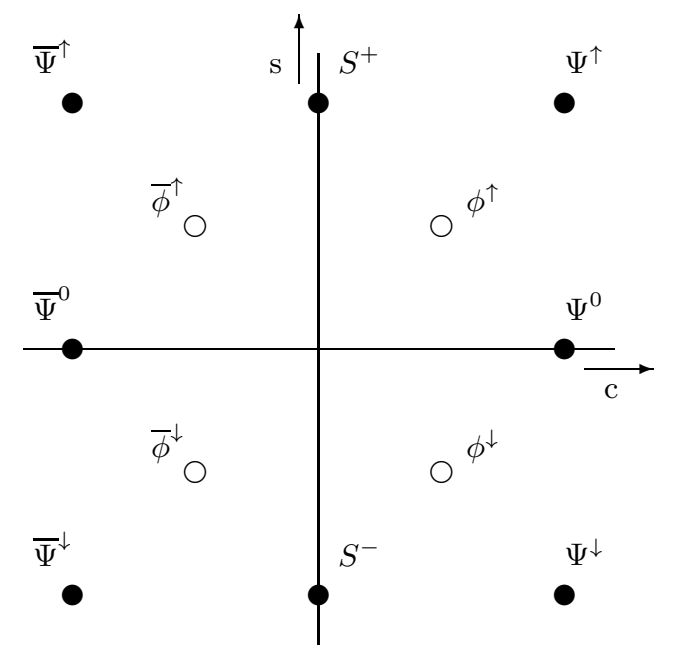

FIG. 11: Roots and weights of the algebra $S O(5)$. The condensate operators $\Psi$ are associated to roots (filled symbols) and the fundamental excitations $\phi$ correspond to the weights of the spinor representation (open symbols).

$S O(5)$ is a rank 2 Lie algebra, which contains mutually commuting $S O(3)$ and $U(1)$ Lie subalgebras, which we can identify with the symmetries under $S O(3)_{\text {spin }}$ and number conservation. In these subalgebras, the generators are respectively $S^{+}, S^{-}, S_{z}$ and $c$ respectively. According to the CFT-qH correspondence ${ }^{17}$, we can also obtain the quantum Hall state wavefunctions as correlators in the chiral part of a CFT, in which the particles (bosons) should be represented by fields that have Abelian braiding properties. In the present case (and the $S U(4)_{k}$ case is similar), the bosons correspond simply to a different triplet of current operators of the $S O(5)_{k}$ affine Kac-Moody algebra. Thus the wavefunction, now in spin components, can be written

$$
\begin{array}{r}
\tilde{\Psi}\left(\left\{z_{i}\right\}\right)=\lim _{z_{\infty} \rightarrow \infty} z_{\infty}^{N / k}\left\langle e^{-i N \varphi_{c} / \sqrt{k}}\left(z_{\infty}\right)\right. \\
\left.J_{\alpha_{1}}\left(z_{1}\right) \cdots J_{\alpha_{N}}\left(z_{N}\right)\right\rangle,
\end{array}
$$

with $J_{\alpha}(\alpha=\downarrow, 0, \uparrow)$ an $S O(3)_{\text {spin }}$ triplet of currents in the affine Lie algebra, that carry $U(1)$ charge +1 . The currents are shown in the $S O(5)$ weight lattice (fig. 11), as $\Psi_{\uparrow}, \Psi_{0}, \Psi_{\downarrow}$. The operator whose position tends to $\infty$ represents a background charge, such that the total $U(1)$ charge of the operators is zero (as it must be in order that the correlator be nonzero). The currents can be expressed as

$$
J_{\alpha}(z)=\psi_{\alpha}(z) e^{i \vec{\beta}_{\alpha} \cdot \vec{\varphi} / \sqrt{k}}(z)
$$

where $\psi_{\alpha}$ is a parafermion field, of conformal weight $1-1 / k$ for the long roots and $1-1 / 2 k$ for the short roots. The vertex operator $e^{i \vec{\beta}_{\alpha} \cdot \vec{\varphi} / \sqrt{k}}(z)$ contains the two free boson fields $\varphi_{c}$ (charge) and $\varphi_{s}$ (spin), with $\vec{\beta}$ the position in the root lattice. For $J_{\alpha}$ these are $\vec{\beta}_{\uparrow}=(1,1)$, $\vec{\beta}_{0}=(1,0), \vec{\beta}_{\downarrow}=(1,-1)$.

The parafermions simplify when we specialize to the case $k=1$, where they reduce to the identity operator for 
the long roots ( $\Psi_{\uparrow}$ and $\Psi_{\downarrow}$, in particular) and a Majorana fermion for the short roots $\left(\Psi_{0}\right)$. The correlator can then be readily written down, as the correlation functions for Majorana fermions is well known:

$$
\left\langle\psi\left(z_{1}\right) \cdots \psi\left(z_{n}\right)\right\rangle=\operatorname{Pf}\left(\frac{1}{z_{i}-z_{j}}\right) .
$$

This reproduces the $S O(5)_{1}$ wavefunction, in the same way as for the spinless Moore-Read state $\underline{\underline{17}}$.

We note that the same $S O(5)_{k}$ algebra was used in another construction 45 , which was for a system of spin$1 / 2$ particles. The present case differs in that the physical $S O(3)_{\text {spin }}$ symmetry is embedded differently in $S O(5)$, because of the different spin of the underlying particles.

Wavefunctions for zero-energy states containing quasiholes can also be written down as chiral correlators, which now contain vertex operators for primary fields of the $S O(5)_{k}$ algebra that represent the quasiholes. For the $k=1$ case, these contain (in the scalar field plus Majorana fermion language) a spin field for the Majorana fermion, and give rise to quasihole wavefunctions analogous to those for the Moore-Read state ${ }^{17.41}$.

\section{Composite fermions}

Alternative QH states to the rather exotic series in the previous two subsections can be constructed by applying conventional methods to spin- 1 bosons. One such approach, as in the case of scalar bosons, is to map the bosons onto composite fermions, by attaching (say) one vortex to each boson. These fermions see a reduced effective magnetic field, and one can construct an incompressible state when an integer number of Landau levels in the effective magnetic field is filled with all three components. This construction gives states with filling factors $\nu=3 p /(3 p \pm 1)$, which are $S U(3)_{\text {spin }}$ singlets.

One can interpret the Moore-Read state as the $p$-wave pairing of composite fermions $\frac{17}{17}$. In this case, $p$-wave $S O(3)$-singlet pairing is possible (in contrast to the spin$1 / 2$ case) and indeed, we have seen that the $S O(5)_{1}$ state can be interpreted this way. In the $S U(3)$ symmetric case at $\nu=1$, no 2-particle $S U(3)$-singlet pairing is possible and there are two options for the system. One is to form a Fermi liquid, the other is to spontaneously break the symmetry and form $(p, q)=(2,0)$ pairs. Note that this last possibility includes the $S O(3)$ singlet and thus can be continuously connected to the $S O(5)_{1}$ state.

\section{Vortex lattices without polar order, and nematic QH liquids}

The earlier discussion of QH liquid states focused on singlets under $S O(3)_{\text {spin }}$, with short range spin correlations. It is interesting to wonder also if QH liquids with some form of spin ordering could occur. One possibility would be a ferromagnetic QH liquid. Such states can be easily written down, by using any wavefunction for a $\mathrm{QH}$ state of spinless bosons, with all the boson spins in the $\alpha=\uparrow$ state (or a global spin rotation of this). One might expect these to occur in the ferromagnetic $\left(c_{2}<0\right)$ part of region II, but in fact we see no sign of them: leaving aside the skyrmion textures in the BEC at low $L$, at larger $L$ all ground states are spin singlets. We note that for spin- $1 / 2$ electrons, spin-polarized states can occur, e.g. at $\nu=1$, even for spin-independent interaction, due to exchange effects. However, the exchange effects are presumably different for bosons.

A more feasible-looking possibility is $\mathrm{QH}$ states with polar spin order, perhaps in the antiferromagnetic region $c_{2}>0$. In the regime at large $\gamma$ where mean-field theory predicts the Abrikosov vortex lattice, the spin-order is polar. In the polar state, the vector condensate can be written as $\left\langle\psi_{\mu}\right\rangle=e^{i \varphi} n_{\mu}$, with $\varphi$ the phase and $\hat{n}$ a real vector. In Abrikosov lattice, the magnitude of the vector $\hat{n}$ and the phase $\varphi$ vary to give a triangular lattice of vortices. We can now imagine that quantum fluctuations destroy either part of the order (restoring either the phase or the spin-rotation symmetry) without the other. When the $U(1)$ and translational symmetry that are broken in the vortex lattice are restored, the ground state is a $\mathrm{QH}$ fluid. For large quantum fluctuations one might expect that the $\mathrm{QH}$ liquid has restored $S U(2)_{\text {spin }}$ symmetry. However, the two transitions at which these symmetries are restored are independent, and the transitions could in principle occur in either sequence as we go to smaller $\nu$. The intermediate phase in which spin symmetry is restored but not the phase would be a vortex lattice in a boson paired state, a BEC of boson pairs. This would be characterized by having a nonzero expectation value of $\sum_{\mu} \psi_{\mu}(z) \psi_{\mu}(z)$, which is invariant under $\psi \rightarrow-\psi$. (Such a vortex lattice would also be possible with vortices containing a half-unit of vorticity each, instead of integers as we assume otherwise, and this might be reached by restoring symmetry in the $\pi$-disclination lattice state.)

The other possible sequence of transitions would be that in which the intermediate phase is a $\mathrm{QH}$ liquid with restored translational and phase symmetry, but still has the polar order, which breaks $S U(2)_{\text {spin. }}$. The singleboson expectation $\left\langle\psi_{\mu}(z)\right\rangle$ would be zero, but if we look at the composite operator $\psi_{\mu}^{\dagger}(z) \psi_{\mu^{\prime}}(z)$, this can have an expectation value. This matrix has a trace equal to the density, which is uniform by assumption. The traceless Hermitian matrix obtained by subtracting off the trace contains antisymmetric and symmetric parts. The (imaginary) antisymmetric part corresponds to a spin-1 irreducible tensor that is simply the spin density, which is assumed to be zero here. The (real) symmetric part corresponds to a spin-2 irreducible tensor. This is the order parameter of a polar or nematic state, which represents a vector $\hat{n}$, but is invariant under $\hat{n} \rightarrow-\hat{n}$, so it parametrizes $S^{2} / Z_{2}=R P^{2}$. Trial wavefunctions for these nematic quantum Hall states can be written down 
as those for scalar bosons, times a spin state such as $\alpha=0$ for all bosons, or as a spin-rotation of this.

It would not be surprising if such nematic QH liquids occurred in the phase diagram at large $\gamma$, now that the corresponding (polar Abrikosov) vortex lattices are known to be present. In finite size, the ground state would always be low $\operatorname{spin}^{33}, S=0$ or 1 , and there need be no transition separating it from a state at the same $L$, $S$ with short-range correlations, such as the $S O(5)_{1}$ state at $g_{0}=0$. Thus the appearance of such nematic order in a QH fluid of spin-1 bosons in the thermodynamic limit cannot yet be ruled out.

\section{E. Numerical results}

To examine how well the proposed states describe the true ground states, we have performed exact diagonalization of small systems. In the regime $L \leq N$, we have used both the disc and sphere geometries. As we have seen, these results differ somewhat. But when looking at fast rotation, however, where the filling factor is of order 1 , the system is spread out into a pancake. It makes sense to focus attention on the interior of the disc and avoid edge effects. This can be done by using an edgeless geometry such as the sphere or torus. Here we will be interested in the ground states in which (unlike the work earlier in this paper) we find the ground states without constraining $L$. QH liquid ground states will usually then show up as $\tilde{L}=0$ states. At finite sizes on the sphere, such ground states that form a sequence of sizes tending to a particular filling factor $\nu$ in the thermodynamic limit lie on a sequence of the form $N_{v}=N / \nu-s^{22}$. Here $s$ is known as the shift, and its appearance is connected with the coupling of the particles to the curvature of the sphere. The value of $s$ depends on the liquid state, not only on $\nu . N_{v}$ can be obtained from the angular momentum on the disc as $L=N N_{v} / 2($ all states have $\tilde{L}=0)$. That is, when the states in the $\tilde{\Psi}$ notation are written for the sphere, one can take $N_{v}$ as small as possible, so that $N_{v}$ equals the highest power of any $z_{i}$ appearing in the wavefunction (see Sec. III). For QH ground states, this will usually mean that $\tilde{L}=0$. For the $S U(4)_{k}$ ground states, we have $N_{v}=4 N /(3 k)-2$, while for the $S O(5)_{k}$ ground states $N_{v}=N / k-2$. We will compare the results of numerical solution for the ground states with these series of trial states.

The $S U(4)_{k}$ states with $k=N / 3$ are the exact ground states for $c_{2}=0$ at $N_{v}=2$ on the sphere. It is not surprising that they are eigenstates, because they are the only $S U(3)$ singlet states, as in the case of $L=N$ for the disc.

As a further test for the $S U(4)_{k}$ states, we have looked at sizes $\left(N, N_{v}\right)$ at which such a ground state could lie for $k>1$. Since $N$ must be divisible by $3 k$, such sizes increase rapidly even for $k=2$. The next case after the trivial BTC for $k=2$ is $N=12, N_{v}=6$. Here it turns out that the overlap-squared of the exact ground state for $c_{2}=0$ with the trial state is ${ }^{46}\left|\left\langle S U(4)_{2} \mid G . S .\right\rangle\right|^{2}=$ 0.915226 .

The $S O(5)_{1}$ state was shown to be an exact ground state for $c_{0}>0, g_{0}=0$. The higher members of this series, however, had a vanishing overlap with the ground states throughout the phase diagram.

As a further test of our proposed wavefunctions, we have performed calculations for torus geometries. On the torus, one simply has $\nu=N / N_{v}$ for the finite size sequence of ground states that tend to a fluid of filling factor $\nu$ in the thermodynamic limit. We saw in the mean field analysis of the skyrmion lattice, that the lattice can only be observed when the number of flux quanta is a multiple of three. However, at low filling factors, we expect to see quantum Hall states at $\nu=3 k / 4$. To be able to observe these, $N_{v}$ has to be a multiple of 4 . Unfortunately, this implies torus sizes which are too large to observe both the quantum liquids and the skyrmion lattice.

To see if the proposed wave-functions are good candidates, we are therefore forced to look at tori which frustrate the mean field skyrmion lattice. The cases we considered are $N_{v}=3,4,6$. For $N_{v}=4$, we find that the ground states are exactly given by the $S U(4)_{k}$ series. However, as for the BTC states on the sphere, this is due to the fact that the trial ground states, which are $S U(3)_{\text {spin }}$ singlet states, span the space of all $S U(3)_{\text {spin }}$ singlets on the torus, which has dimension equal to eq. (25), the degeneracy of $S U(4)_{k}$ torus ground states. Clearly this must be independent of the geometry of the torus (described by $\tau$ ), and we verified this in some cases.

In fig. 12 we have plotted the particle-hole excitation gap

$$
\Delta(N)=N\left(\frac{E(N-1)}{N-1}+\frac{E(N+1)}{N+1}-2 \frac{E(N)}{N}\right),
$$

where $E(N)$ is the ground state energy for $N$ particles, for $N_{v}=4$. In the thermodynamic limit, this quantity will exhibit upward peaks at filling factors that corrrespond to incompressible states. We performed the diagonalization for total $S O(3)$ spin $S=0,1,2$.

For $N_{v}=6$ (figure 13), we focussed on the state at $\nu=3 / 2, N=9$, which corresponds to $k=2$. We have calculated the overlap-squared with the $S U(4)_{2}$ state to be 0.939804 . Another feature in the $N_{v}=6$ plot is the state at $\nu=1(N=6)$. This could be a precursor to a paired composite fermion state; however, the overlap with the $S O(5)_{1}$ state was small.

\section{F. The boundaries of region II}

The behavior at the phase boundaries $\mathrm{I}-\mathrm{A} / \mathrm{II}$ and $\mathrm{I}-$ B/II (see figure 1) deserves special attention. At the boundary I-B/II, where $\gamma=c_{2} / c_{0}=-1$, the Hamiltonian simplifies as $g_{2}=0$ and only the contact interaction which projects onto the spin-singlet channel remains. As a result of this, large degeneracies occur. For example, 


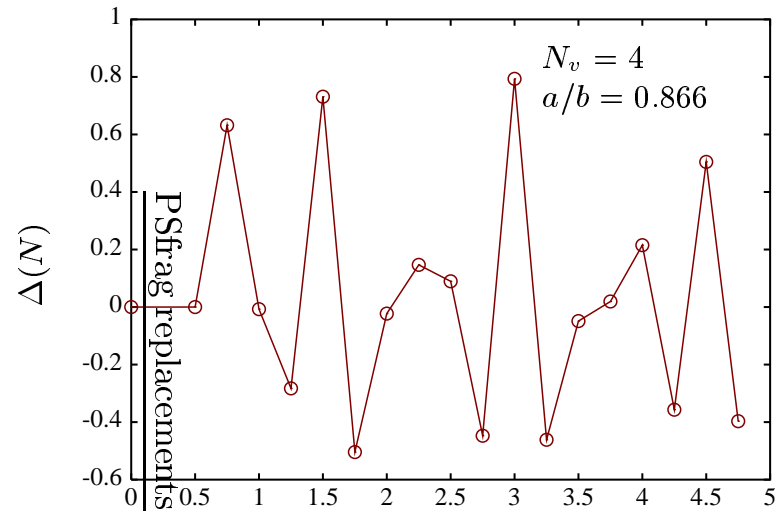

FIG. 12: Particle-hole excitation gap $\Delta(N)$ versus $\nu$, for $N_{v}=4$, in a rectangular geometry, $a / b=\sqrt{3} / 2$. The peaks can be interpreted as an indication of incompressibility of the corresponding states. For $N=3,6,9$ we verified that the ground states, which are degenerate, are exactly the $S U(4)_{k}$ quantum Hall trial states with $k=1,2,3$, respectively.

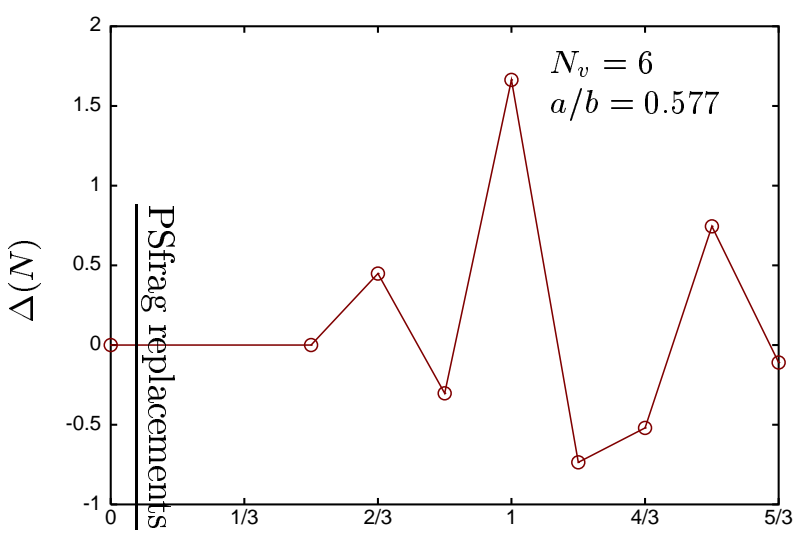

FIG. 13: Particle-hole excitation gap $\Delta(N)$ versus $\nu$, for $N_{v}=$ 6 , in a rectangular geometry, $a / b=\sqrt{3} / 3$.

all fully-polarized states $(S=N)$ have zero energy. We have not obtained analytic expressions for these degeneracies. That they are not due to the specific geometry was observed on the torus. The zero energy states are not sensitive to changes in the geometry. As examples of these degeneracies, we have in figure 14 tabulated the $\tilde{L}$, $S$ quantum numbers of the zero energy states for $N=6$, $N_{v}=2$ and for $N=5, N_{v}=3$ on the sphere, and for $N=6$ particles in the disc geometry.

\section{CONCLUSION}

In this paper we have studied the phase diagram of spin-1 bosons in a rotating trap, within the LLL approximation, using a variety of techniques (numerical diagonalization, mean field theory, and analytical constructions). We concentrated on certain regimes. These were

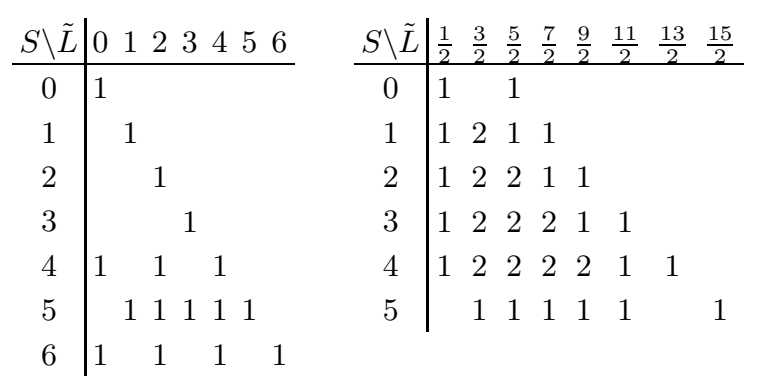

\begin{tabular}{c|rrrrrrrr}
$S \backslash L$ & 0 & 1 & 2 & 3 & 4 & 5 & 6 & $\ldots$ \\
\hline 0 & & & & & & 1 & $\ldots$ \\
1 & & & & & 1 & 1 & $\ldots$ \\
2 & & & & 1 & 1 & 3 & $\ldots$ \\
3 & & & & 1 & 1 & 3 & 5 & $\ldots$ \\
4 & & 1 & 1 & 3 & 4 & 8 & $\ldots$ \\
5 & & 1 & 1 & 2 & 3 & 5 & 6 & $\ldots$ \\
6 & 1 & 1 & 1 & 2 & 2 & 4 & $\ldots$
\end{tabular}

FIG. 14: Degeneracies of zero-energy ground states on the sphere at $\gamma=-1$ for $N=6, N_{v}=2$ (top left), $N=5$, $N_{v}=3$ (top right) and $N=6, N_{v}=\infty$. All multiplicities refer to highest weight states of the orbital $S O(3)$ symmetry.

(i) low rotation, such that the angular momentum $L$ is less than or equal to the particle number $N$, where the system is beginning to contain some vorticity; (ii) higher rotation, where the bulk of the fluid accommodates vorticity and is occupied by a lattice of (possibly coreless) vortices, which we considered as infinite periodic structures; (iii) the quantum Hall regime, in which the vortex lattices are replaced by translationally-invariant quantum fluids, which we considered in edgeless geometries (unfortunately, for spin-1 bosons the finite size restrictions are here very severe). The transition regions between these regimes, namely that in which the system contains a small number (larger than two) of vortices, and that at the critical filling factor at which the vortex lattices are replaced by the quantum liquids, were not considered. The results show a rich variety of phases as the interaction parameters, especially the ratio of the coefficients of spin-dependent and spin-independent interaction terms, are varied. The results obtained here, especially those at lower rotations which should be more easily accessible, should motivate further experiments to rotate spin-1 bosons with unbroken spin-rotation symmetry.

\section{A. Acknowledgements}

We thank Ed Rezayi for collaboration in an early stage of this study and for discussions; we acknowledge discussions with T.-L. Ho, E.J. Mueller, R. Shankar. This research was supported by the Netherlands Organisation for Scientific Research, NWO, and the Foundation FOM 
of the Netherlands (J.W.R., F.J.M.v.L. and K.S.), and by the NSF under grant no. DMR-02-42949 (N.R.).

\section{APPENDIX A: CLASSIFICATION OF TOPOLOGICAL DEFECTS}

Here we continue the discussion of the topological classification of defects or excitations.

In Sec. I we explained the appearance of two types of ordered BECs for spin-1 bosons. In these the order is constant in space. More generally, a condensate will prefer to have the same type of order locally at almost all points in order to lower the energy, but there are types of excitations in which the order as described by a point in the order parameter manifold can vary in space. Excitations of this type that are stable under continuous deformations of the order (known as topological defects) can be classified by methods from topology. One type is those in which the order breaks down (possible, the density goes to zero) at a point in space (we consider two space dimensions here). These are classified by the fundamental or first homotopy group $\pi_{1}$ of the manifold. Such objects exist for example in the case of a one-component (scalar) condensate, where the order parameter manifold is the circle $S^{1}$. For vector condensates, an example is the polar state, for which $\pi_{1}\left(S^{1} \times S^{2} / \mathbb{Z}_{2}\right)=\mathbb{Z}$, the group of integers. Because these involve the phase of the condensate (related to the $S^{1}$ part of the manifold) winding as one encircles the singular point, these objects in both examples are vortices that are relevant when vorticity is forced into the system. In the polar state, the vortices of the smallest vorticity carry a half unit of vorticity (in the usual units), because of the $\mathbb{Z}_{2}$ divided out, and are also referred to as $\pi$-disclinations. For the case of the ferromagnetic state, $\pi_{1}(S O(3))=\mathbb{Z}_{2}$, so that two nontrivial vortices can annihilate one another ${ }^{5}$.

The other main type of topological defect is sometimes called a coreless vortex or skyrmion. In these the order exists and varies within the order parameter manifold everywhere in space. The topological classification requires identifying points at infinity, as if space were a sphere. For trivial boundary conditions (those that allow the constant ordered ground state), the topological defects are classified by the second homotopy group $\pi_{2}$ of the manifold. This is $\mathbb{Z}$ in the polar case, and trivial in the ferromagnetic case. However, if we wish to classify the vortices that carry the nonzero vorticity in a rotating condensate, then the boundary conditions must be modified to allow a nonzero vorticity on the sphere (this modification has of the form of the Dirac string familiar for a magnetic monopole). In the polar case, the presence of net vorticity may force vortices into the ground state. Whether the ground state contains $\pi$-disclinations, or vortices of larger vorticity each, depends on the detailed energetics, but one should note that as the total vorticity is an integer $N_{v}$, the number of $\pi$-disclinations (more generally, the number of vortices that carry half- integer vorticity) must be even. On the other hand, the defects above that are classified by $\pi_{2}$ carry no vorticity. For the ferromagnetic case, there are coreless vortices or skyrmions which are topologically-nontrivial textures in the order and carry nonzero vorticity quantized in integers. We will discuss these configurations in more detail below.

We now come to the borderline case in which the interaction is spin-independent $\left(S U(3)_{\text {spin }}\right.$ invariant $), c_{2}=0$. This is a useful starting point for small $\gamma$ also. The discussion is easily generalized to the case of an $n$ component order parameter with an $S U(n)$ invariant interaction. In such a case, the target space is described by a complex $n$-component vector of unit length, proportional to the expectation value of the boson operator, which lies in $U(n) / U(n-1) \equiv S^{2 n-1}$. For $n>1$, $\pi_{1}=\pi_{2}=0$, so this space has neither point-singular vortices nor skyrmions without vorticity. For $n=1$, there are the well-known point-singular vortices with one unit of vorticity each, and for $n>1$ the presence of nonzero vorticity on the sphere induces skyrmions with integer vorticity in the condensate, as we will now see.

In more detail, the Bose condensate on a sphere with vorticity present is described by the expectation value of the field operator $\left\langle\psi_{\mu}(z)\right\rangle$ as $z$ ranges over the sphere. Thus the possible condensates form the space of sections of a complex vector bundle. Such bundles are classified topologically (for each number of components $n>0$ ) by their first Chern class, which is simply the (integer) number of vortices $N_{v}$ that we have been using, or the number of flux quanta in the monopole at the center of the sphere ${ }^{22}$. Restricting the bosons to the LLL means considering only the "holomorphic" sections of the same bundles. The LLL mean field theory performed in Section $\nabla$ simply finds such holomorphic sections of lowest mean-field energy. Condensates in which there are $N_{v}$ vortices at which the density (magnitude squared of the condensate) vanishes always exist; simply take the condensate entirely in one spin component. The question we will pursue here is the existence of coreless vortices or skyrmions with non-zero vorticity, in which the condensate is nonzero at all points on the sphere. These correspond to nonsingular configurations of the order as described above.

The nicest configurations of all, which serve to illustrate the most elementary skyrmions, are those in which the density is uniform over the sphere. [Note that for repulsive spin-independent interactions with $S U(n)_{\text {spin }}$ symmetry, the ground state makes the density as uniform as possible.] It is convenient to study these in the LLL in terms of their components $b_{m \mu}, \mu=1, \ldots, n$, and $m=0, \ldots, N_{v}$. These components form a matrix $B$, with $m$ labelling rows and $\mu$ labelling columns. The density is uniform if and only if

$$
B B^{\dagger} \propto I
$$

where $I$ is the identity matrix (that is, $B^{\dagger}$ is proportional to an isometry). Solutions to this condition exist only 
when $0 \leq N_{v} \leq n-1$. For $N_{v}>n-1$, skyrmions will still occur, but the density will not be completely uniform.

We now specialize to $n=3$ again, and consider the special cases $N_{v}=1$ and $N_{v}=2$ for which uniform density condensates exist. For $N_{v}=1, m=0,1$ only, so $B$ is a $2 \times 3$ matrix. A solution of eq. A1 for $B^{\dagger}$ is a complex number times an isometry of $\mathbf{C}^{2}$ into $\mathbf{C}^{3}$. Thus this means picking a two-dimensional subspace of the spin space $\mathbf{C}^{3}$. Notice that $S U(2)_{\text {orb }}$ acts by multiplication of $B$ on the left, while $S U(3)_{\text {spin }}$ acts by multiplication (by the transpose) on the right, and the phase symmetry under $U(1)$ acts on either side of $B$. Then the full space of solutions to eq. (A1), for fixed mean particle number, is parametrized by $S U(2)_{\text {orb }} \times S U(3)_{\text {spin }} \times$ $U(1) /[S U(2) \times U(1)]$, where the denominator represents the subgroup of $S U(3)_{\text {spin }}$ which has the same action as $S U(2)_{\text {orb }} \times U(1)$ on a particular solution $B$. This manifold is equivalent to $S U(3) / \mathbb{Z}_{3}$. If we consider a quantum state in which bosons condense in one condensate in this family, then we can analyze it in terms of $N, \tilde{L}, \tilde{L}_{z}$, and $S U(3)_{\text {spin }}$ quantum numbers $(p, q)$. It is easy to see that there can be no $S U(3)_{\text {spin }}$ singlets for $N_{v}=1$ as construction of such a singlet requires the use of three distinct orbitals. Thus the broken-symmetry states cannot be averaged over $S U(3)$ spin rotations to produce an $S U(3)$ singlet (the closest one can get would be the BDC of Section [IV] and we believe that state should be interpreted in this way). Nonetheless, we have shown the existence of configurations with single units of vorticity. We have now analyzed the space of solutions with uniform density using the full $S U(3)_{\text {spin }}$ symmetry group. For general $c_{2} \neq 0$, this symmetry is broken. In this case, the form of the lowest-energy solution depends on the energetics, and the manifold of lowest energy condensates (orbit of the solution under the broken symmetries) is a submanifold of that above with a lower dimension that depends on which solution is chosen. Results of this analysis have been given in Sec. V

Similarly, for $N_{v}=2$, there are three orbitals, and the matrix $B$ is now $3 \times 3$. We see directly from eq. (A1) that a solution for $B$ is proportional to a unitary matrix, and the manifold of solutions is therefore $U(3)$. In terms of the symmetries present for $c_{2}=0$ (i.e. using $S U(3)_{\text {spin }}$ symmetry), this manifold arises as $S U(3)_{\text {spin }} \times S O(3)_{\text {orb }} \times U(1) / S O(3) \times \mathbb{Z}_{3}$. In this case the $S O(3)$ in the denominator, which is the unbroken subgroup of the spin and orbital symmetries, has to be embedded into $S U(3)$ as the group of $3 \times 3$ orthogonal matrices. The $U(1)$ transformations cannot be removed using the orbital or spin symmetry groups.

The uniform-density skyrmions for $N_{v}=2$ and $c_{2} \neq 0$ can be found by using a careful choice of basis. For the ferromagnetic case $c_{2}<0$, one expects that at each point on the sphere there should be a nonzero (in fact, largest possible) spin density, though its orientation varies over the sphere. If we use the basis of single-particle $S_{z}$ eigenstates in the sequence $\alpha=\downarrow, 0$, , then one such solution is given by $B \propto \operatorname{diag}(1,-1,1)$. In the particular solution given, the spin density is $\downarrow$ at the north pole $(z=0$ in stereographic coordinates $), \uparrow$ at the south pole $(z \rightarrow \infty)$, and the orientation elsewhere on the sphere can be found by the relation of spin and orbital rotations as described in the previous paragraph. In fact, the spin-density $\langle\vec{S}\rangle$ itself wraps around the sphere, being $(0,0,-1)$ at the north pole, $(0,0,1)$ at the south pole, and in the $x y$ plane at the equator. This solution is the $\ell=1$ solution discussed in Sec. V Other solutions are obtained by multiplication of $B$ by an element of $S O(3) \times U(1)$. The space of these solutions forms the manifold $S O(3)_{\mathrm{spin}} \times S O(3)_{\mathrm{orb}} \times U(1) / S O(3) \simeq$ $S O(3) \times U(1)$, a submanifold of the full $U(3)$ we had before. The solution $B=\operatorname{diag}(0,-1,0)$ we began with here was chosen to be invariant under the diagonal $S O(3)$ subgroup generated by $\overrightarrow{\tilde{L}}+\vec{S}$. The other solutions, obtained by acting with either $S O(3)_{\text {orb }}$ or $S O(3)_{\text {spin }}$, are invariant under a similar subgroup with generators $\overrightarrow{\tilde{L}}$ plus a (fixed) $S O(3)$ rotation of $\vec{S}$. These solutions are very similar to the basic skyrmions for the spin- $1 / 2$ case, which appear in the $\nu=1 \mathrm{QH}$ effect for electrons 35 , even though here for spin 1 they have $N_{v}=2$. In fact those solutions for a condensate of spin-1/2 bosons correspond to the $n=2$ component case with a contact interaction and $S U(2)$ symmetry, as discussed briefly above.

In the antiferromagnetic regime $\gamma>0$ for $N_{v}=2$, one expects a different mean field ground state. As $\gamma \rightarrow 0^{+}$, the ground state should approach a point in a submanifold of the $U(3)$ manifold of uniform density states. Presumably this submanifold is a different one from that for $\gamma<0$, where the solutions always lie in the set we just described. In fact, we expect that the ground states selected at $\gamma \rightarrow 0+$ are those with the lowest spin density at each point on the sphere. At large $\gamma$, the spin order at each point should take the polar form, with vanishing spin density. This presumably cannot happen with a uniform density. One expects the preceding uniform density solutions to persist at small positive $\gamma$, and then (possibly, above a nonzero critical value of $\gamma$ ) for the density to become non-uniform. At large enough $\gamma$, we expect the $N_{v}=2$ solution to contain two polar vortices, each with vorticity one. These correspond to solutions found in $\operatorname{Sec} \nabla$

For each of the manifolds that describe lowest-energy mean field solutions, we can consider the configuration space of a point moving on this manifold, and then quantize this motion (this is known as semiclassical quantization of the collective coordinates). This should reproduce the full space of states for the $N \rightarrow \infty$ limit for these cases $N_{v}=1,2$, but we will not go into these details here. 
1 C.J. Myatt et al., Phys. Rev. Lett. 78, 586 (1997).

2 M.D. Barrett et al., Phys. Rev. Lett. 87, 010404 (2001).

3 D.M. Stamper-Kurn et al., Phys. Rev. Lett. 80, 2027 (1998); J. Stenger et al., Nature 396, 345 (1998); H-J. Miesner et al., Phys. Rev. Lett. 82, 2228 (1999).

4 J.W. Reijnders, F.J.M. van Lankvelt, K. Schoutens and N. Read, Phys. Rev. Lett. 89, 120401 (2002).

5 T.-L. Ho, Phys. Rev. Lett. 81, 742 (1998).

${ }^{6}$ F. Zhou, Phys. Rev. Lett. 87, 80401 (2001).

7 S.K. Yip, Phys. Rev. Lett. 83, 4677 (1999).

8 T. Isoshima and K. Machida, Phys. Rev. A 66, 23602 (2002).

9 T. Mizushima, K. Machida and T. Kita, Phys. Rev. Lett. 8930401 (2002).

10 T. Mizushima, K. Machida and T. Kita, Phys. Rev. A 66, $53610(2002)$

11 K.W. Madison et al., Phys. Rev. Lett. 84, 806 (2000); J.R. Abo-Shaeer et al., Science 292, 476 (2001).

12 D.A. Butts and D.S. Rokhsar, Nature 397, 327 (1999).

13 T. Kita, T. Mizushima, K. Machida, Phys. Rev. A66, 061601 (2002).

14 N.R. Cooper, N.K. Wilkin, and J.M.F. Gunn, Phys. Rev. Lett. 87, 120405 (2001).

15 N. Read and E. Rezayi, Phys. Rev. B 59, 8084 (1999).

16 R. B. Laughlin, Phys. Rev. Lett. 50, 1395 (1983).

17 G. Moore and N. Read, Nucl. Phys. B 360, 362 (1991).

18 S.M. Girvin and A.H. MacDonald, Phys. Rev. Lett. 58, 1252 (1987).

19 N. Read, Phys. Rev. Lett. 62, 86 (1989).

20 N. Read and D. Green, Phys. Rev. B 61, 10267 (2000).

21 N. Regnault and Th. Jolicoeur, cond-mat/0212477

22 F.D.M. Haldane, Phys. Rev. Lett. 51, 605 (1983).

${ }^{23}$ F.D.M. Haldane, Phys. Rev. Lett. 55, 2059 (1985).

24 T. Ohmi and K. Machida, J. Phys. Soc. Jpn. 67, 1822 (1998).

25 N.K. Wilkin, J.M.F. Gunn and R.A. Smith, Phys. Rev. Lett. 80, 2265 (1998).

26 T.-L. Ho and S.K. Yip, Phys. Rev. Lett. 84, 4031 (2000).
27 T.-L. Ho and E.J. Mueller, Phys. Rev. Lett. 89, 050401 (2002).

28 We thank Ed Rezayi for providing numerical data that prompted our derivation of this result.

29 N.K. Wilkin and J.M.F. Gunn, Phys. Rev. Lett. 84, 6 (2000).

30 G.F. Bertsch and T. Papenbrock, Phys. Rev. Lett. 83, 5412 (1999).

31 R.A. Smith and N.K. Wilkin, cond-mat/0005230

32 P. Nozières and D. Saint James, J. Phys. 43, 1133 (1982).

33 P.W. Anderson, Basic Notions of Condensed Matter Physics (Benjamin-Cimmings, Menlo Park, CA, 1984), Chapter 2.

34 J.-P. Martikainen, A. Collin and K.-A. Suominen, Phys. Rev. A66, 53604 (2002).

35 S.L. Sondhi, A. Karlhede, S.A. Kivelson and E.H. Rezayi, Phys. Rev. B 47, 16419 (1993).

${ }^{36}$ E. Mueller and T.-L. Ho, Phys. Rev. Lett. 88, 80403 (2002).

37 R. Shankar, private communication.

38 B. Paredes, P. Zoller and J.I. Cirac, Phys. Rev. A 66, 033609 (2002).

39 A. Cappelli, L.S. Georgiev, I.T. Todorov, Nucl. Phys. B599, 499-530 (2001).

${ }^{40}$ K. Schoutens, E. Ardonne, F.J.M. van Lankvelt, in Proc. of the NATO Advanced Research Workshop "Statistical Field Theories" Como (Italy), June 18-23 2001, A. Cappelli and G. Mussardo, eds. (Kluwer Academic Publishers, 2002), 305, (cond-mat/0112379).

41 N. Read and E. Rezayi, Phys. Rev. B 54, 16864 (1996).

42 V. Gurarie and E. Rezayi, Phys. Rev. B 61, 5473 (2000).

43 E. Ardonne, N. Read, E. Rezayi and K. Schoutens, Nucl. Phys. B 607, 549 (2001).

44 E. Ardonne, J. Phys. A 35, 447 (2002).

45 E. Ardonne, F.J.M. van Lankvelt, A.W.W. Ludwig, and K. Schoutens, Phys. Rev. B 65, 041305 (2002).

46 E. Rezayi, private communication. 